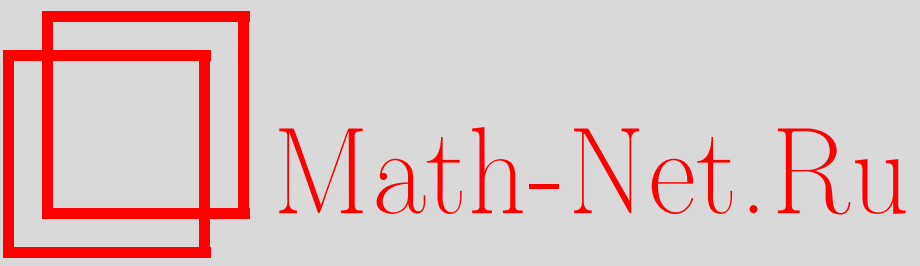

М. А. Васильев, С. Ф. Прокушкин, Когомологии токов произвольного спина в пространстве $\mathrm{AdS}_{3}, T M \Phi, 2000$, том 123, номер 1, 3-25

DOI: https://doi.org/10.4213/tmf1942

Использование Общероссийского математического портала Math-Net.Ru подразумевает, что вы прочитали и согласны с пользовательским соглашением

http://www.mathnet.ru/rus/agreement

Параметры загрузки:

IP : 54.198 .64 .247

26 апреля 2023 г., 15:28:04 


\author{
ТЕОРЕТИЧЕСКАЯ \\ И МАТЕМАТИЧЕСКАЯ \\ ФИЗИКА \\ Том 123, № 1 \\ апрель, 2000
}

(C) 2000 г.

М. А. Васильев* , С. Ф. Прокушкин*

\title{
КОГОМОЛОГИИ ТОКОВ ПРОИЗВОЛЬНОГО СПИНА В ПРОСТРАНСТВЕ $\mathrm{AdS}_{3}$
}

\begin{abstract}
Изучаются сохраняющиеся токи произвольных целых и полуцелых спинов, построенные из безмассовых скалярных и спинорных полей в пространстве $\mathrm{AdS}_{3}$. Показано, что 2-формы, дуальные сохраняюшимся токам в $\mathrm{AdS}_{3}$, являются точными в классе бесконечных разложений по высшим производным полей материи с коэффициентами, содержащими отрицательные степени космологической постоянной. Это свойство не имеет аналога в плоском пространстве и может быть связано с голографичностью пространств анти-де Ситтера. "Улучшения" физических токов описаны как тривиальный локальный класс когомологий токов. Определен комплекс токов спина $s\left(T^{s}, \mathcal{D}\right)$ и найдена группа когомологий $H^{1}\left(T^{s}, \mathcal{D}\right)=\mathbb{C}^{2 s+1}$.
\end{abstract}

\section{1. ВВЕДЕНИЕ}

В последнее время сильно возрос интерес к геометрии анти-де Ситтера (AdS) в связи с гипотезой Малдасены [1] о дуальности между теорией гравитации в пространстве AdS и конформной теорией на его границе [2, 3]. Голографическая гипотеза утверждает, что эти два типа теорий эквивалентны. В то же время геометрия AdS играет очень важную роль в теории калибровочных полей высших спинов (краткий обзор содержится в работе [4]), поскольку взаимодействия калибровочных полей высших спинов содержат отрицательные степени космологической постоянной [5]. Теория калибровочных полей высших спинов может рассматриваться [4] как кандидат на роль максимально симметричной фазы теории струн.

Случай группового многообразия $\mathrm{AdS}_{3}$ является специальным и интересен во многих отношениях. В частности, двумерные модели на границе $\mathrm{AdS}_{3}$ являются конформными [6]. С точки зрения теории высших спинов спешифическое свойство моделей в трехмерном пространстве-времени состоит в том, что калибровочные поля высших спинов являются нераспространяющимися, в полной аналогии с обычными гравитационными и янг-миллсовскими полями Черна-Саймонса. Тем не менее калибровочные симметрии высших спинов остаются нетривиальными подобно гравитационным (спин 2) и внутренним (спин 1) симметриям. Токи высших спинов могут быть построены из полей материи спинов 0 и $1 / 2$ и описывают взаимодействия материи посредством калибровочных полей высших спинов.

* Физический институт им. П. Н. Лебедева РАН, Москва, Россия. E-mail: vasiliev@td.lpi.ac.ru; prok@td.lpi.ac.ru 
Схематически уравнения движения в секторе калибровочных полей можно записать в виде

$$
R=J(C ; W)
$$

где $R=d W-W \wedge W-$ кривизны всех спинов $s \geqslant 1$, построенные из потенциала высших спинов $W$, а $C$ - поля материи (точные определения даются в разделе 2). 2-форма $J(C ; W)$, дуальная трехмерному векторному полю сохраняюшегося тока, вследствие уравнений движения в секторе полей материи подчиняется закону сохранения

$$
D J(C ; W)=0,
$$

где $D$ - ковариантная производная, соответствующая (бесконечномерной) калибровочной алгебре высших спинов $[7,8]$, т.е. $\delta R=D \delta W, \delta W$ - произвольная вариация калибровочного потенциала высших спинов. Для пертурбативного исследования задачи выберем вакуумное решение $W_{0}$, удовлетворяюшее уравнению

$$
R_{0}=0
$$

и предположим, что $W=W_{0}+W_{1}$, а $C$ имеет первый порядок малости. В тех случаях, когда гравитация включена в модель, как это имеет место в калибровочных теориях высших спинов, $W_{0}$ отлично от нуля и описывает фоновую геометрию. В низшем неисчезающем порядке из (1.1) получаем

$$
R_{1} \equiv D_{0} W_{1}=J_{2}\left(C^{2}\right)
$$

где дифференциал $D_{0}$ построен из $W_{0}$, а $J_{2}\left(C^{2}\right)$ есть билинейная по $C$ часть $J\left(C ; W_{0}\right)$. Закон сохранения (1.2) требует, чтобы выполнялось условие

$$
D_{0} J_{2}\left(C^{2}\right)=0
$$

на свободных уравнениях движения для полей материи.

Нелинейная система уравнений движения, описывающая калибровочные взаимодействия высших спинов для полей материи спинов 0 и $1 / 2$ в $\mathrm{AdS}_{3}$ во всех порядках по взаимодействию, была сформулирована как для безмассовых [9], так и для массивных [10] полей материи. Интересным свойством предложенных уравнений, которое было обнаружено в работе [10], является сушествование потока, генерируюшего отображение полной нелинейной системы в свободную. Это отображение является нелинейной заменой полевых переменных, имеюшей вид бесконечного ряда по старшим производным полей материи, и в общем случае нелокально. Коэффициенты в таких разложениях содержат отрищательные степени космологической постоянной (чем выше производная поля материи, тем старше отрицательная степень космологической постоянной) и, следовательно, не допускают плоского предела. Мы называем такие разложения по высшим производным псевдолокальными, чтобы отличать их от нелокальных выражений, которые невозможно представить в виде рядов по старшим производным.

Сравнение результатов [10] с (1.4) показьвает, что такое переопределение полей сушествует в нетривиальной модели в том случае, если

$$
J_{2}\left(C^{2}\right)=D_{0} U\left(C^{2}\right)
$$


где $U$ - некоторый псевдолокальный функционал от полей материи. Когомологическая интерпретация с дифференциалом $D_{0}$, рассматриваемым как дифференциал де $\mathrm{Pa-}$ ма, очевидна, поскольку $D_{0}^{2}=R_{0}=0$. Действительно, из (1.5) следует, что ток $J_{2}\left(C^{2}\right)$ должен быть замкнутым на свободных уравнениях движения для полей материи, тогда как (1.6) означает, что он точен в классе псевдолокальных функционалов.

Этот факт уже был продемонстрирован в работе [10] для тока спина 2, где мы нашли псевдолокальный функционал $U$ для тензора энергии-импульса, построенного из безмассового скалярного поля. Мы обобшаем этот результат на токи произвольных целых и полуцелых спинов, которые содержат минимальное возможное число пространственно-временных производных. Анализ токов произвольно высокого спина сильно упрощается благодаря использованию формализма производящих функций, развиваемого в данной статье. Этот формализм основан на так называемой развернутой формулировке релятивистских уравнений [11], которая позволяет исследовать задачу алгебраически, автоматически учитьвая тот факт, что задача рассматривается на массовой оболочке.

Токи, которые точны с локальным функционалом $U$, содержашим конечное число производных от полей материи, воспроизводят “улучшения", т.е. такие модификации токов, которые сохраняются тривиально. Новым результатом является то, что "истинные" токи также могут рассматриваться как “улучшения" в классе псевдолокальных разложений. Это выглядит многообещающе, поскольку в связи с гипотезой голографичности соответствующие переопределения полей могут приводить к нетривиальным эффектам на границе.

Статья организована следующим образом. В разделе 2 собраны некоторые факты, касающиеся уравнений движения калибровочных полей Черна-Саймонса высших спинов и развернутой формулировки уравнений движения для безмассовых полей материи спинов 0 и $1 / 2$ в $\mathrm{AdS}_{3}$. В разделе 3 предложен формализм производящих функций для описания дифференциальных форм, билинейных по полям материи. Используя этот метод, в разделе 4 мы формулируем AdS-комплекс на массовой оболочке и в разделе 5 изучаем его когомологии - когомологии токов. В разделе 6 мы обсуждаем плоский предел.

\section{2. ПОЛЯ ВЫСШИХ СПИНОВ И ПОЛЯ МАТЕРИИ В $\mathrm{AdS}_{3}$}

Трехмерные калибровочные поля высших спинов описываются $[8,9]$ 1-формой $W=$ $d x^{\mu} W_{\mu}(y, \psi \mid x)$, зависящей от координат пространства-времени $x^{\mu}(\mu=0,1,2)$, вспомогательных коммутируюших спинорных переменных $y_{\alpha}$ (индексы $\alpha, \beta, \gamma=1,2$ поднимаются и опускаются симплектической формой $\epsilon_{\alpha \beta}=-\epsilon_{\beta \alpha}, \epsilon_{12}=\epsilon^{12}=1, A^{\alpha}=$ $\left.\epsilon^{\alpha \beta} A_{\beta}, A_{\alpha}=A^{\beta} \epsilon_{\beta \alpha}\right)$ и центрального инволютивного элемента $\psi, \psi^{2}=1$ :

$$
W_{\mu}(y, \psi \mid x)=\sum_{n=0}^{\infty} \frac{1}{2 i n !}\left[\omega_{\mu, \alpha_{1} \ldots \alpha_{n}}(x)+\lambda \psi h_{\mu, \alpha_{1} \ldots \alpha_{n}}(x)\right] y^{\alpha_{1}} \ldots y^{\alpha_{n}}
$$

Постоянный параметр $\lambda$ будет отождествлен ниже с обратным радиусом $\mathrm{AdS}_{3}$.

Калибровочная алгебра высших спинов есть супералгебра Ли, построенная посредством (анти)коммутаторов из ассоциативной алгебры, образованной элементами вида (2.1), с законом умножения

$$
(f * g)(y, \psi)=\frac{1}{(2 \pi)^{2}} \int d^{2} u d^{2} v \exp \left(i u_{\alpha} v^{\alpha}\right) f(y+u, \psi) g(y+v, \psi),
$$


где переменные интегрирования $u_{\alpha}$ и $v_{\alpha}$ - двухкомпонентные спиноры (в соответствии с (2.1) бозонно-фермионная четность идентифицируется с четностью по вспомогательным переменным $y$ ). Этот закон умножения дает специальную реализацию алгебры Вейля, $\left[y_{\alpha}, y_{\beta}\right]_{*}=2 i \epsilon_{\alpha \beta}$. Напряженность поля есть $[7,8]$

$$
R(y, \psi \mid x)=d W(y, \psi \mid x)-W(y, \psi \mid x) * \wedge W(y, \psi \mid x)
$$

а уравнения движения для калибровочных полей Черна-Саймонса высших спинов с материальным источником имеют вид (1.1).

Элемент $\psi$ введен для того, чтобы калибровочная алгебра высших спинов была полупростой $(h s(2) \oplus h s(2)$ в обозначениях [7]). При этом простые компоненты выделяются проекторами $P_{ \pm}=(1 \pm \psi) / 2$, аналогично алгебре изометрии пространства $\mathrm{AdS}_{3} \quad o(2,2) \sim s p(2) \oplus s p(2)$. Последняя отождествляется с подалгеброй алгебры $h s(2) \oplus h s(2)$, образованной элементами

$$
L_{\alpha \beta}=\frac{1}{2 i} y_{\alpha} y_{\beta}, \quad P_{\alpha \beta}=\frac{1}{2 i} y_{\alpha} y_{\beta} \psi,
$$

поэтому мы отождествляем $o(2,2)$-компоненты поля $W(y, \psi \mid x)(2.1)$ с гравитационными 1-формой лоренцевой связности $\omega^{\alpha \beta}(x)=d x^{\mu} \omega_{\mu}{ }^{\alpha \beta}(x)$ и 1-формой триады $h^{\alpha \beta}(x)=$ $d x^{\mu} h_{\mu}{ }^{\alpha \beta}(x)$. Поскольку $\mathrm{AdS}_{3}$ алгебра $o(2,2)$ - подалгебра алгебры высших спинов в трехмерном пространстве-времени, то требование, чтобы вакуумное значение поля $W(y, \psi \mid x)$ было отлично от нуля только в секторе спина 2 , задает непротиворечивый анзац. Тогда уравнение $R_{0}=0$ эквивалентно условиям нулевой кривизны для $o(2,2)$

$$
\begin{aligned}
& d \omega_{\alpha \beta}=\omega_{\alpha \gamma} \wedge \omega_{\beta}{ }^{\gamma}+\lambda^{2} h_{\alpha \gamma} \wedge h_{\beta}{ }^{\gamma}, \\
& d h_{\alpha \beta}=\omega_{\alpha \gamma} \wedge h_{\beta}{ }^{\gamma}+\omega_{\beta \gamma} \wedge h_{\alpha}{ }^{\gamma} .
\end{aligned}
$$

Для метрической интерпретации триада $h_{\nu},{ }^{\alpha \beta}$ должна быть невырожденной, т.е. должна сушествовать обратная триада $h^{\nu}, \alpha \beta$,

$$
h_{\nu,}{ }^{\alpha \beta} h^{\nu}, \gamma \delta=\frac{1}{2}\left(\delta_{\gamma}^{\alpha} \delta_{\delta}^{\beta}+\delta_{\delta}^{\alpha} \delta_{\gamma}^{\beta}\right)
$$

(мы используем нормировку, принятую в работе [11]). Тогда (2.6) сводится к условию нулевого кручения, которое выражает лоренцеву связность $\omega_{\nu},{ }^{\alpha \beta}$ через триаду $h_{\nu},{ }^{\alpha \beta}$, а условие (2.5) означает, что $\mathcal{R}_{\alpha \beta}=-\lambda^{2} h_{\alpha \gamma} \wedge h_{\beta}{ }^{\gamma}$, где $\mathcal{R}_{\alpha \beta}$ - 2-форма тензора Римана. Тем самым уравнения (2.5) и (2.6) описывают пространство-время $\mathrm{AdS}_{3}$ с радиусом $\lambda^{-1}$, т.е. геометрия $\mathrm{AdS}_{3}$ возникает через решение вакуумного уравнения (1.3).

Безмассовые уравнения Клейна-Гордона и Дирака в $\mathrm{AdS}_{3}$ имеют вид

$$
\square C=\frac{3}{2} \lambda^{2} C, \quad h^{\mu}{ }_{, \alpha}^{\beta} \nabla_{\mu} C_{\beta}=0
$$

для бозонного поля $C(x)$ спина 0 и фермионного поля $C_{\alpha}(x)$ спина $1 / 2$. Здесь $\square=$ $\nabla^{\mu} \nabla_{\mu}$, где $\nabla_{\mu}$ - полная ковариантная производная с симметричными символами Кристоффеля, определяемыми через метрический постулат

$$
\nabla_{\mu} h_{\nu}{ }^{\alpha \beta}=0
$$


Мировые индексы $\mu, \nu$ поднимаются и опускаются с помошью метрического тензора $g_{\mu \nu}=h_{\mu}{ }^{\alpha \beta} h_{\nu, \alpha \beta}$.

Для анализа когомологий токов наиболее удобна "развернутая" формулировка [11] уравнений (2.8) в виде некоторых условий ковариантного постоянства. Для этого вводится бесконечный набор симметричных мультиспиноров $C_{\alpha_{1} \ldots \alpha_{n}}$ для всех $n \geqslant 0$ (следуя [12], мы подразумеваем, что по индексам, обозначенным одинаковой буквой, проведена полная симметризация, и используем обозначение $C_{\alpha(n)}=C_{\alpha_{1} \ldots \alpha_{n}}$ в тех случаях, когда важно лишь число индексов). Как показано в [11], бесконечная цепочка уравнений

$$
D^{L} C_{\alpha(n)}=\frac{i}{2}\left[h^{\beta \gamma} C_{\beta \gamma \alpha(n)}-\lambda^{2} n(n-1) h_{\alpha \alpha} C_{\alpha(n-2)}\right],
$$

где $D^{L}$ - фоновый лоренц-ковариантный дифференциал,

$$
D^{L} C_{\alpha(n)}=d C_{\alpha(n)}+n \omega_{\alpha}^{\gamma} C_{\gamma \alpha(n-1)},
$$

эквивалентна уравнениям (2.8) для компонент низших рангов $C$ и $C_{\alpha}$ и некоторым связям, которые выражают высшие мультиспиноры через высшие пространственно-временные производные от $C$ и $C_{\alpha}$ следуюшим образом:

$$
\begin{aligned}
C_{\alpha(2 n)}(x) & =(-2 i)^{n} h^{\nu_{1}}{ }_{, \alpha \alpha} h^{\nu_{2}}{ }_{, \alpha \alpha} \ldots h^{\nu_{n}}{ }_{, \alpha \alpha} \nabla_{\nu_{1}} \nabla_{\nu_{2}} \ldots \nabla_{\nu_{n}} C(x), \\
C_{\alpha(2 n+1)}(x) & =(-2 i)^{n} h^{\nu_{1}}{ }_{, \alpha \alpha} h^{\nu_{2}}{ }_{, \alpha \alpha} \ldots h^{\nu_{n}}{ }_{, \alpha \alpha} \nabla_{\nu_{1}} \nabla_{\nu_{2}} \ldots \nabla_{\nu_{n}} C_{\alpha}(x) .
\end{aligned}
$$

Заметим, что для мультиспиноров $\nabla_{\mu} C_{\alpha(n)}=D_{\mu}^{L} C_{\alpha(n)}$.

Следуя [11], введем производяшую функцию

$$
C(y, \psi \mid x)=\sum_{n=0}^{\infty} \frac{1}{n !}\left(\lambda^{-1} \psi\right)^{\left[\frac{n}{2}\right]} C_{\alpha_{1} \ldots \alpha_{n}}(x) y^{\alpha_{1}} \ldots y^{\alpha_{n}}=\lambda^{\frac{1}{2} \pi(C)} \widetilde{C}\left(\lambda^{-\frac{1}{2}} y, \psi \mid x\right),
$$

где $[n+a]=n \forall n \in \mathbb{Z}$ и $0 \leqslant a<1$, а бозонно-фермионная четность $\pi(C)=0$ для четных и $\pi(C)=1$ для нечетных функций $C(y)$. Уравнения (2.10) можно переписать в виде [11]

$$
D^{L} C(y, \psi)=\frac{i \lambda}{2} \psi h^{\alpha \beta}\left[\frac{\partial}{\partial y^{\alpha}} \frac{\partial}{\partial y^{\beta}}-y_{\alpha} y_{\beta}\right] C(y, \psi),
$$

где $D^{L}=d-\omega^{\alpha \beta} y_{\alpha}\left(\partial / \partial y^{\beta}\right)$.

Заметим, что в определение (2.13) введены отрицательные степени $\lambda$ для того, чтобы уравнения (2.10) следовали из (2.14) или, что эквивалентно, чтобы коэффициенты в (2.12) не зависели от $\lambda$. Формула (2.13) демонстрирует то общее свойство, что высшие производные в теории появляются вместе с отрицательными степенями космологической постоянной.

Поля $C_{\alpha_{1} \ldots \alpha_{n}}$ отождествляются со всеми нетривиальными на массовой оболочке производными полей материи согласно (2.12). То, что система рассматривается на массовой оболочке, неявно обеспечивается фактом полной симметричности мультиспиноров $C_{\alpha_{1} \ldots \alpha_{n}}$. Это позволяет использовать компоненты $C_{\alpha_{1} \ldots \alpha_{n}}$ вместо явного введения производных от полей материи. 
Теперь рассмотрим функцию $F\left[C_{\alpha(n)}(x)\right]$ всех компонент $C_{\alpha_{1} \ldots \alpha_{n}}(x)$ в некоторой фиксированной точке $x$. При этом предполагается, что функция $F$ не содержит производных по координатам пространства-времени $x^{\nu}$ и, следовательно, выглядит как локальная функция полей материи. Однако здесь надо проявить аккуратность, поскольку если выполняются уравнения (2.10), то верно (2.12). Мы будем называть функцию $F\left[C_{\alpha(n)}\right]$ псевдолокальной, если она является бесконечным разложением по полевым переменным $C_{\alpha(n)}(x)$, и локальной, если $F$ - полином с конечным числом ненулевых слагаемых.

Используя производящие функции $C(y, \psi \mid x)$, это можно переформулировать следуюшим образом. Пусть $F(C \mid x)$ - некоторый функционал от производяшей функции $C(y, \psi \mid x)$ в некоторой фиксированной точке пространства-времени $x$. Согласно (2.12) его пространственно-временная локальность эквивалентна на массовой оболочке локальности в $y$-пространстве. Действительно, из (2.14) следует, что производные по спинорным переменным являются в некотором смысле квадратным корнем из пространственно-временных производных (что очевидно также из (2.12)).

Уравнение (1.4) для системы высших спинов в трехмерном пространстве-времени имеет вид (везде далее мы используем обозначение $D$ вместо $D_{0}$ )

$$
D W_{1}(y, \psi \mid x)=J\left(C^{2}\right)(y, \psi \mid x)
$$

с фоновым AdS-ковариантным дифференциалом

$$
D=D^{L}-\lambda \psi h^{\alpha \beta} y_{\alpha} \frac{\partial}{\partial y^{\beta}}=d-\left(\omega^{\alpha \beta}+\lambda \psi h^{\alpha \beta}\right) y_{\alpha} \frac{\partial}{\partial y^{\beta}} .
$$

Тот факт, что 1-формы $\omega^{\alpha \beta}(x)$ и $h^{\alpha \beta}(x)$ подчиняются уравнениям $(2.5)$ и $(2.6)$, обеспечивает равенство $D^{2}=0$. Таким образом, нашей задачей является исследование когомологий оператора $D(2.16)$. Очевидно, $D$ коммутирует с оператором Эйлера $N=$ $y^{\alpha}\left(\partial / \partial y^{\alpha}\right)$, собственные значения которого отождествляются со спином $s$,

$$
N=2(s-1)
$$

Следовательно, задача должна исследоваться для разных спинов независимо.

Сохраняющиеся токи произвольного целого спина в четырехмерном пространстве Минковского рассматривались в работе [13]. Для случая $d=2$ были построены сохраняюшиеся токи высших спинов [14], также некоторые токи спинов выше 2 недавно обсуждались в работе [15].

В случае $\mathrm{AdS}_{3}$ сохраняющиеся токи любого целого спина $s \geqslant 1$, построенные из двух безмассовых скалярных полей $C, C^{\prime}$ или двух безмассовых спинорных полей $C_{\alpha}, C_{\alpha}^{\prime}$ 
имеют вид

$$
\begin{aligned}
J_{\mu, \alpha(2 s-2)}^{(s)}\left(C, C^{\prime}\right)= & \sum_{k=0}^{s-2} \frac{2(-1)^{k}}{(2 k+1) !(2 s-2 k-3) !} h_{\mu},{ }^{\gamma \gamma} C_{\gamma \alpha(2 k+1)} C_{\gamma \alpha(2 s-2 k-3)}^{\prime}+ \\
& +\sum_{k=0}^{s-1} \frac{(-1)^{k}}{(2 k) !(2 s-2 k-2) !} h_{\mu},{ }^{\gamma \gamma} \times \\
& \times\left[C_{\gamma \gamma \alpha(2 k)} C_{\alpha(2 s-2 k-2)}^{\prime}-C_{\alpha(2 k)} C_{\gamma \gamma \alpha(2 s-2 k-2)}^{\prime}\right] \\
J_{\mu, \alpha(2 s-2)}^{(s)}\left(C_{\alpha}, C_{\alpha}^{\prime}\right)= & \sum_{k=0}^{s-1} \frac{2(-1)^{k+1}}{(2 k) !(2 s-2 k-2) !} h_{\mu},{ }^{\gamma \gamma} C_{\gamma \alpha(2 k)} C_{\gamma \alpha(2 s-2 k-2)}^{\prime}+ \\
& +\sum_{k=0}^{s-2} \frac{(-1)^{k}}{(2 k+1) !(2 s-2 k-3) !} h_{\mu},{ }^{\gamma \gamma} \times \\
& \times\left[C_{\gamma \gamma \alpha(2 k+1)} C_{\alpha(2 s-2 k-3)}^{\prime}-C_{\alpha(2 k+1)} C_{\gamma \gamma \alpha(2 s-2 k-3)}^{\prime}\right]
\end{aligned}
$$

Суперток любого полуцелого спина $s \geqslant 3 / 2$, построенный из безмассовых скаляра $C$ и спинора $C_{\alpha}^{\prime}$, имеет вид

$$
\begin{gathered}
J_{\mu, \alpha(2 s-2)}^{(s)}\left(C, C_{\alpha}^{\prime}\right)=\sum_{k=0}^{s-\frac{3}{2}}\left\{\frac{2(-1)^{k}}{(2 k+1) !(2 s-2 k-3) !} h_{\mu},{ }^{\gamma \gamma} C_{\gamma \alpha(2 k+1)} C_{\gamma \alpha(2 s-2 k-3)}^{\prime}+\right. \\
\left.+\frac{(-1)^{k}}{(2 k) !(2 s-2 k-2) !} h_{\mu}{ }^{\gamma \gamma}\left[C_{\gamma \gamma \alpha(2 k)} C_{\alpha(2 s-2 k-2)}^{\prime}-C_{\alpha(2 k)} C_{\gamma \gamma \alpha(2 s-2 k-2)}^{\prime}\right]\right\} .(2.2 .2
\end{gathered}
$$

Сохраняюшиеся токи низших спинов имеют вид

$$
\begin{aligned}
J_{\mu}^{(1)}\left(C, C^{\prime}\right)= & h_{\mu}{ }^{\gamma \gamma}\left(C_{\gamma \gamma} C^{\prime}-C C_{\gamma \gamma}^{\prime}\right), \quad J_{\mu}^{(1)}\left(C_{\alpha}, C_{\alpha}^{\prime}\right)=h_{\mu},{ }^{\gamma \gamma} C_{\gamma} C_{\gamma}^{\prime}, \\
J_{\mu, \alpha}^{(3 / 2)}\left(C, C_{\alpha}^{\prime}\right)= & h_{\mu}{ }^{\gamma \gamma}\left(C_{\gamma \gamma} C_{\alpha}^{\prime}-C C_{\gamma \gamma \alpha}^{\prime}+2 C_{\gamma \alpha} C_{\gamma}^{\prime}\right) \\
J_{\mu, \alpha \alpha}^{(2)}\left(C, C^{\prime}\right)= & \frac{1}{2} h_{\mu},{ }^{\gamma \gamma}\left(C_{\gamma \gamma} C_{\alpha \alpha}^{\prime}-C C_{\gamma \gamma \alpha \alpha}^{\prime}-\right. \\
& \left.-C_{\gamma \gamma \alpha \alpha} C^{\prime}+C_{\alpha \alpha} C_{\gamma \gamma}^{\prime}+4 C_{\gamma \alpha} C_{\gamma \alpha}^{\prime}\right) .
\end{aligned}
$$

Все эти токи локальны, поскольку каждый из них содержит конечное число членов (т.е. высших производных (2.12)). Те же выражения остаются справедливыми и в плоском пределе, $\nabla_{\mu} \rightarrow \partial_{\mu}$ в (2.12).

\section{3. ПРОИЗВОДЯЩИЕ ФУНКЦИИ}

Для решения когомологической задачи для токов произвольных спинов разовьем метод, оперирующий производящими функциями (2.13), а не отдельными мультиспинорами. 
Общая лоренш-ковариантная пространственно-временная 1-форма спина $s=n / 2+1$, билинейная по двум различным материальным полям $C$ и $C^{\prime}$ и по их производным, нетривиальным на массовой оболочке, имеет вид

$$
\begin{aligned}
\Phi_{\alpha(n)}\left(C, C^{\prime} \mid x\right)= & \sum_{k+l=n-2} \sum_{m=0}^{\infty} a(k, l, m) h_{\alpha \alpha} C_{\alpha(k)}^{\beta(m)}(x) C_{\alpha(l) \beta(m)}^{\prime}(x)+ \\
& +\sum_{k+l=n-1} \sum_{m=0}^{\infty}\left[b_{1}(k, l, m) h_{\alpha}^{\gamma} C_{\gamma \alpha(k)}^{\beta(m)}(x) C_{\alpha(l) \beta(m)}^{\prime}(x)+\right. \\
& \left.+b_{2}(k, l, m) h_{\alpha}^{\gamma} C_{\alpha(k)}^{\beta(m)}(x) C_{\gamma \alpha(l) \beta(m)}^{\prime}(x)\right]+ \\
& +\sum_{k+l=n} \sum_{m=0}^{\infty}\left[e_{1}(k, l, m) h^{\gamma \gamma} C_{\gamma \gamma \alpha(k)}^{\beta(m)}(x) C_{\alpha(l) \beta(m)}^{\prime}(x)+\right. \\
& +e_{2}(k, l, m) h^{\gamma \gamma} C_{\gamma \alpha(k)}^{\beta(m)}(x) C_{\gamma \alpha(l) \beta(m)}^{\prime}(x)+ \\
& \left.+e_{3}(k, l, m) h^{\gamma \gamma} C_{\alpha(k)}^{\beta(m)}(x) C_{\gamma \gamma \alpha(l) \beta(m)}^{\prime}(x)\right]
\end{aligned}
$$

где $a(k, l, m), b_{1,2}(k, l, m)$ и $e_{1,2,3}(k, l, m)$ - произвольные константы, а $h_{\alpha \alpha}-1$-форма триады. Вводя

$$
\Phi(y, \psi \mid x)=\Phi_{\alpha_{1} \ldots \alpha_{n}}(\psi \mid x) y^{\alpha_{1}} \ldots y^{\alpha_{n}}
$$

можно эквивалентно переписать формулу (3.2) в виде

$$
\begin{aligned}
& \Phi(y, \psi \mid x)=h_{\alpha \alpha} \frac{1}{(2 \pi)^{2}} \oint d r \oint d s \oint \tau^{-2} d \tau \int d^{2} u d^{2} v \exp \left\{\frac{i}{\tau}\left(u_{\gamma} v^{\gamma}\right)\right\} \times \\
& \quad \times C(u-r y, \psi \mid x) C^{\prime}(v+s y, \psi \mid x)\left[f_{1}(r, s, \tau) y^{\alpha} y^{\alpha}+f_{2}(r, s, \tau) y^{\alpha} u^{\alpha}+\right. \\
& \left.\quad+f_{3}(r, s, \tau) y^{\alpha} v^{\alpha}+f_{4}(r, s, \tau) u^{\alpha} u^{\alpha}+f_{5}(r, s, \tau) u^{\alpha} v^{\alpha}+f_{6}(r, s, \tau) v^{\alpha} v^{\alpha}\right] .
\end{aligned}
$$

Здесь $r, s$ и $\tau$ - комплексные переменные, а $u_{\alpha}$ и $v_{\alpha}(\alpha=1,2)$ - спинорные переменные. Величины $f_{i}(r, s, \tau), i=1, \ldots, 6$, являются полиномами по $r^{-1}$ и $s^{-1}$ и формальными рядами по $\tau^{-1}$ вида

$$
\begin{aligned}
& f_{1}(r, s, \tau)=\sum_{\substack{0<k, l<p, p<\infty}} \sum_{m=1}^{\infty} f_{1}(k, l, m) r^{-k} s^{-l} \tau^{-m} \\
& f_{2,3}(r, s, \tau)=\sum_{\substack{0<k, l<p, m=2 \\
p<\infty}} \sum_{m, 3}^{\infty} f_{2,3}(k, l, m) r^{-k} s^{-l} \tau^{-m} \\
& f_{4,5,6}(r, s, \tau)=\sum_{\substack{0<k, l<p, p<\infty}} \sum_{m=3}^{\infty} f_{4,5,6}(k, l, m) r^{-k} s^{-l} \tau^{-m} \text {. }
\end{aligned}
$$

Контурные интегрирования нормированы следующим образом:

$$
\oint \tau^{-n} d \tau=\delta_{n}^{1}
$$


Гауссовы интегрирования по $u_{\alpha}$ и $v_{\alpha}$ должны быть выполнены до контурных интегрирований.

Подставляя (2.13) в виде

$$
\begin{aligned}
C(u-r y, \psi \mid x)= & \sum_{n, m=0}^{\infty} \frac{1}{n ! m !}\left(\lambda^{-1} \psi\right)^{\left[\frac{n+m}{2}\right]} \times \\
& \times C_{\alpha(n) \beta(m)}(x) u^{\alpha_{1}} \ldots u^{\alpha_{n}}(-r)^{m} y^{\beta_{1}} \ldots y^{\beta_{m}} \\
C^{\prime}(v+s y, \psi \mid x)= & \sum_{n, m=0}^{\infty} \frac{1}{n ! m !}\left(\lambda^{-1} \psi\right)^{\left[\frac{n+m}{2}\right]} \times \\
& \times C_{\alpha(n) \beta(m)}^{\prime}(x) v^{\alpha_{1}} \ldots v^{\alpha_{n}} s^{m} y^{\beta_{1}} \ldots y^{\beta_{m}}
\end{aligned}
$$

в (3.3) и выполняя элементарные интегрирования, приходим к (3.1) со следуюшими коэффициентами:

$$
\begin{aligned}
a(k, l, m) & =\frac{(-1)^{k+m} i^{m}}{k ! l ! m !}\left(\lambda^{-1} \psi\right)^{\left[\frac{k+m}{2}\right]+\left[\frac{l+m}{2}\right]} f_{1}(k+1, l+1, m+1), \\
b_{1}(k, l, m) & =\frac{(-1)^{k+m} i^{m+1}}{k ! l ! m !}\left(\lambda^{-1} \psi\right)^{\left[\frac{k+m+1}{2}\right]+\left[\frac{l+m}{2}\right]} f_{3}(k+1, l+1, m+2), \\
b_{2}(k, l, m) & =\frac{(-1)^{k+m+1} i^{m+1}}{k ! l ! m !}\left(\lambda^{-1} \psi\right)^{\left[\frac{k+m}{2}\right]+\left[\frac{l+m+1}{2}\right]} f_{2}(k+1, l+1, m+2), \\
e_{1}(k, l, m) & =\frac{(-1)^{k+m+1} i^{m}}{k ! l ! m !}\left(\lambda^{-1} \psi\right)^{\left[\frac{k+m}{2}\right]+\left[\frac{l+m}{2}\right]+1} f_{6}(k+1, l+1, m+3) \\
e_{2}(k, l, m) & =\frac{(-1)^{k+m} i^{m}}{k ! l ! m !}\left(\lambda^{-1} \psi\right)^{\left[\frac{k+m+1}{2}\right]+\left[\frac{l+m+1}{2}\right]} f_{5}(k+1, l+1, m+3), \\
e_{3}(k, l, m) & =\frac{(-1)^{k+m+1} i^{m}}{k ! l ! m !}\left(\lambda^{-1} \psi\right)^{\left[\frac{k+m}{2}\right]+\left[\frac{l+m}{2}\right]+1} f_{4}(k+1, l+1, m+3)
\end{aligned}
$$

Таким образом, формула (3.3) действительно описывает общую лоренц-ковариантную 1-форму, билинейную по полям материи. Из этих выражений мы видим, что формула (3.3) воспроизводит пространственно-локальное выражение, если все коэффициенты $f_{i}$ содержат конечное число членов в (3.4)-(3.6), и псевдолокальное, если некоторые из разложений по отрицательным степеням $\tau$ бесконечны.

Как будет показано ниже, на практике наиболее удобны следующие представления дифференциальных форм $\Phi_{n}(x)$ ранга $n=0,1,2,3$ :

$$
\begin{aligned}
& \Phi_{0}(y, \psi \mid x)=\psi \frac{1}{(2 \pi)^{2}} \oint \frac{d z}{z} \oint \frac{d \bar{z}}{\bar{z}} \oint \frac{d \tau}{\tau^{2}} \int d^{2} q d^{2} \bar{q} \exp \left\{-\frac{1}{2 \tau}\left(q_{\gamma} \bar{q}^{\gamma}\right)\right\} \times \\
& \times C\left[\frac{1}{2}(q+\bar{q})-\frac{1}{2 i}(z-\bar{z}) y, \psi \mid x\right] C^{\prime}\left[\frac{1}{2 i}(q-\bar{q})+\frac{1}{2}(z+\bar{z}) y, \psi \mid x\right] E_{0}(z, \bar{z}, \tau) \\
& \Phi_{1}(y, \psi \mid x)=h_{\alpha \alpha} \frac{1}{(2 \pi)^{2}} \oint d z \oint d \bar{z} \oint \tau^{-2} d \tau \int d^{2} q d^{2} \bar{q} \exp \left\{-\frac{1}{2 \tau}\left(q_{\gamma} \bar{q}^{\gamma}\right)\right\} \times \\
& \times C\left[\frac{1}{2}(q+\bar{q})-\frac{1}{2 i}(z-\bar{z}) y, \psi \mid x\right] C^{\prime}\left[\frac{1}{2 i}(q-\bar{q})+\frac{1}{2}(z+\bar{z}) y, \psi \mid x\right] \times
\end{aligned}
$$




$$
\begin{aligned}
& \times\left\{R_{1}(z, \bar{z}, \tau) y^{\alpha} y^{\alpha}+\frac{1}{2 \tau \bar{z}} W_{1}(z, \bar{z}, \tau) y^{\alpha} \bar{q}{ }^{\alpha}+\frac{1}{2 \tau z} \bar{W}_{1}(z, \bar{z}, \tau) y^{\alpha} q^{\alpha}+\right. \\
&\left.+\frac{1}{2 \tau^{2} \bar{z}^{2}} Y_{1}(z, \bar{z}, \tau) \bar{q}^{\alpha} \bar{q}^{\alpha}+\frac{1}{2 \tau^{2} z^{2}} \bar{Y}_{1}(z, \bar{z}, \tau) q^{\alpha} q^{\alpha}+\frac{1}{2 \tau^{2} z \bar{z}} V_{1}(z, \bar{z}, \tau) q^{\alpha} \bar{q}^{\alpha}\right\} \\
& \Phi_{2}(y, \psi \mid x)=-\frac{\lambda}{2} \psi h_{\alpha \beta} \wedge h^{\beta}{ }_{\alpha} \times \\
& \times \frac{1}{(2 \pi)^{2}} \oint d z \oint d \bar{z} \oint \tau^{-2} d \tau \int d^{2} q d^{2} \bar{q} \exp \left\{-\frac{1}{2 \tau}\left(q_{\gamma} \bar{q}^{\gamma}\right)\right\} \times \\
& \times C\left[\frac{1}{2}(q+\bar{q})-\frac{1}{2 i}(z-\bar{z}) y, \psi \mid x\right] C^{\prime}\left[\frac{1}{2 i}(q-\bar{q})+\frac{1}{2}(z+\bar{z}) y, \psi \mid x\right] \times \\
& \times\left\{R_{2}(z, \bar{z}, \tau) y^{\alpha} y^{\alpha}+\frac{1}{2 \tau \bar{z}} W_{2}(z, \bar{z}, \tau) y^{\alpha} \bar{q}{ }^{\alpha}+\frac{1}{2 \tau z} \bar{W}_{2}(z, \bar{z}, \tau) y^{\alpha} q^{\alpha}+\right. \\
&\left.+\frac{1}{2 \tau^{2} \bar{z}^{2}} Y_{2}(z, \bar{z}, \tau) \bar{q}^{\alpha} \bar{q}^{\alpha}+\frac{1}{2 \tau^{2} z^{2}} \bar{Y}_{2}(z, \bar{z}, \tau) q^{\alpha} q^{\alpha}+\frac{1}{2 \tau^{2} z \bar{z}} V_{2}(z, \bar{z}, \tau) q^{\alpha} \bar{q}^{\alpha}\right\} \\
& \Phi_{3}(y, \psi \mid x)=-\frac{\lambda^{2}}{12} h_{\alpha \beta} \wedge h_{\gamma}^{\beta} \wedge h^{\gamma \alpha} \times \\
& \quad \times \frac{1}{(2 \pi)^{2}} \oint \frac{d z}{z} \oint \frac{d \bar{z}}{\bar{z}} \oint \frac{d \tau}{\tau^{2}} \int d^{2} q d^{2} \bar{q} \exp \left\{-\frac{1}{2 \tau}\left(q_{\gamma} \bar{q}{ }^{\gamma}\right)\right\} \times \\
& \quad \times C\left[\frac{1}{2}(q+\bar{q})-\frac{1}{2 i}(z-\bar{z}) y, \psi \mid x\right] C^{\prime}\left[\frac{1}{2 i}(q-\bar{q})+\frac{1}{2}(z+\bar{z}) y, \psi \mid x\right] E_{3}(z, \bar{z}, \tau)
\end{aligned}
$$

Здесь факторы $\psi,-(\lambda / 2) \psi$ и $-\lambda^{2} / 12$ введены для будушего удобства. Нетрудно видеть, что выражения (3.15)-(3.18) воспроизводят произвольные лоренц-ковариантные формы, билинейные по полям материи и их производным, нетривиальным на массовой оболочке.

Пусть $n, \bar{n}$ и $n_{\tau}$ - следующие операторы:

$$
n=z \frac{\partial}{\partial z}, \quad \bar{n}=\bar{z} \frac{\partial}{\partial \bar{z}}, \quad n_{\tau}=\tau \frac{\partial}{\partial \tau}
$$

(мы будем использовать те же обозначения и для их собственных значений). Величины $R_{1,2}(z, \bar{z}, \tau), \quad W_{1,2}(z, \bar{z}, \tau), \quad \bar{W}_{1,2}(z, \bar{z}, \tau), \quad Y_{1,2}(z, \bar{z}, \tau), \bar{Y}_{1,2}(z, \bar{z}, \tau), \quad V_{1,2}(z, \bar{z}, \tau)$ и $E_{0,3}(z, \bar{z}, \tau)$ дают ненулевой вклад в (3.15)-(3.18), если $n, \bar{n}$ и $n_{\tau}$ удовлетворяют ограничениям, указанным в таблице.

\begin{tabular}{|c|c|c|c|}
\hline$R_{1}, R_{2}$ & $n \leqslant-1$ & $\bar{n} \leqslant-1$ & $n_{\tau} \leqslant-1$ \\
\hline$W_{1}, W_{2}$ & $n \leqslant-1$ & $\bar{n} \leqslant 0$ & $n_{\tau} \leqslant-1$ \\
\hline $\bar{W}_{1}, \bar{W}_{2}$ & $n \leqslant 0$ & $\bar{n} \leqslant-1$ & $n_{\tau} \leqslant-1$ \\
\hline$Y_{1}, Y_{2}$ & $n \leqslant-1$ & $\bar{n} \leqslant 1$ & $n_{\tau} \leqslant-1$ \\
\hline $\bar{Y}_{1}, \bar{Y}_{2}$ & $n \leqslant 1$ & $\bar{n} \leqslant-1$ & $n_{\tau} \leqslant-1$ \\
\hline$V_{1}, V_{2}, E_{0}, E_{3}$ & $n \leqslant 0$ & $\bar{n} \leqslant 0$ & $n_{\tau} \leqslant-1$ \\
\hline
\end{tabular}


Вне этих областей коэффициенты не дают вклада, и, следовательно, их значения могут быть фиксированы произвольным образом. В частности, величины $R_{1,2}, W_{1,2}, \bar{W}_{1,2}, \ldots$ определены с точностью до произвольных полиномов по $\tau$

$$
P(\tau)=\sum_{k=0}^{k_{0}} P_{k} \tau^{k} .
$$

Формулы, рассмотренные в этом разделе, имеют смысл для произвольной размерности спиноров (мы не касаемся вопроса о полноте базисных форм типа $h_{\alpha \beta}$ и $h_{\alpha \beta} \wedge h_{\alpha}^{\beta}$ ). Для двухкомпонентных спиноров сушествуют дополнительные соотношения эквивалентности из-за того, что антисимметризация по любым трем двухкомпонентным индексам дает ноль. Этот факт выражается тождеством

$$
a_{\alpha}\left(b_{\beta} c^{\beta}\right)+b_{\alpha}\left(c_{\beta} a^{\beta}\right)+c_{\alpha}\left(a_{\beta} b^{\beta}\right)=0
$$

верным для любых трех коммутируюших двухкомпонентных спиноров $a_{\alpha}, b_{\alpha}$ и $c_{\alpha}$. В результате не все рассматриваемые до сих пор формы являются независимыми. Неоднозначность в добавлении членов, равных нулю вследствие (3.21), может быть выражена в виде некоторых преобразований эквивалентности (калибровочных преобразований) для коэффициентов в (3.3), (3.16) и (3.17). Мы будем называть эти преобразования эквивалентности преобразованиями Фирца.

Чтобы вывести функциональную форму общего преобразования Фирца, удобно переписать (3.16) в виде

$$
\begin{aligned}
\Phi_{1}(y, \psi \mid x)= & h_{\alpha \alpha} \frac{1}{(2 \pi)^{2}} \oint d z \oint d \bar{z} \oint d t \int d^{2} q d^{2} \bar{q} \times \\
& \times \exp \left\{-\frac{t}{2}\left(q_{\gamma} \bar{q}^{\gamma}\right)-\frac{i}{2} z\left(\bar{q} y_{\gamma} y^{\gamma}\right)+\frac{i}{2} \bar{z}\left(y_{\gamma} q^{\gamma}\right)\right\} C\left(\frac{q+\bar{q}}{2}, \psi \mid x\right) \times \\
& \times C^{\prime}\left(\frac{q-\bar{q}}{2 i}, \psi \mid x\right)\left\{f_{1}^{\prime}(z, \bar{z}, t) y^{\alpha} y^{\alpha}+f_{2}^{\prime}(z, \bar{z}, t) y^{\alpha} \bar{q}^{\alpha}+f_{3}^{\prime}(z, \bar{z}, t) y^{\alpha} q^{\alpha}+\right. \\
& \left.+f_{4}^{\prime}(z, \bar{z}, t) \bar{q}^{\alpha} \bar{q}^{\alpha}+f_{5}^{\prime}(z, \bar{z}, t) q^{\alpha} \bar{q}^{\alpha}+f_{6}^{\prime}(z, \bar{z}, t) q^{\alpha} q^{\alpha}\right\} .
\end{aligned}
$$

Выполняя интегрирования по частям по $t, z$ и $\bar{z}$ в $(3.22)$, получаем, что преобразования

$$
\begin{array}{lll}
\delta f_{1}^{\prime}=\partial_{t} \epsilon, & \delta f_{2}^{\prime}=i \partial_{\bar{z}} \epsilon, & \delta f_{3}^{\prime}=-i \partial_{z} \epsilon, \\
\delta f_{3}^{\prime}=\partial_{t} \eta, & \delta f_{5}^{\prime}=i \partial_{\bar{z}} \eta, & \delta f_{6}^{\prime}=-i \partial_{z} \eta, \\
\delta f_{2}^{\prime}=\partial_{t} \phi, & \delta f_{4}^{\prime}=i \partial_{\bar{z}} \phi, & \delta f_{5}^{\prime}=-i \partial_{z} \phi
\end{array}
$$

с произвольными параметрами $\epsilon=\epsilon(z, \bar{z}, t), \eta=\eta(z, \bar{z}, t)$ и $\phi=\phi(z, \bar{z}, t)$ описывают все возможные преобразования Фирца для 1-формы (3.22). Отсюда выводится вид преобразований Фирца в представлениях (3.16) и (3.17)

$$
\begin{aligned}
\delta R_{1,2} & =-\partial_{\tau} \chi_{1,2} \\
\delta W_{1,2} & =-\partial_{\tau} \xi_{1,2}+2 i \bar{n} \chi_{1,2}, \\
\delta \bar{W}_{1,2} & =-\partial_{\tau} \bar{\xi}_{1,2}-2 i n \chi_{1,2} \\
\delta V_{1,2} & =-i\left(n \xi_{1,2}-\bar{n} \bar{\xi}_{1,2}\right) \\
\delta Y_{1,2} & =i(\bar{n}-1) \xi_{1,2} \\
\delta \bar{Y}_{1,2} & =-i(n-1) \bar{\xi}_{1,2}
\end{aligned}
$$


с произвольными параметрами $\chi_{1,2}(z, \bar{z}, \tau), \xi_{1,2}(z, \bar{z}, \tau)$ и $\bar{\xi}_{1,2}(z, \bar{z}, \tau)$.

Отметим, что соотношение

$$
h_{\alpha \beta} \wedge h_{\gamma \delta}=\frac{1}{4}\left(\varepsilon_{\alpha \gamma} h_{\beta \lambda} \wedge h_{\delta}^{\lambda}+\varepsilon_{\beta \gamma} h_{\alpha \lambda} \wedge h_{\delta}^{\lambda}+\varepsilon_{\beta \delta} h_{\alpha \lambda} \wedge h_{\gamma}^{\lambda}+\varepsilon_{\alpha \delta} h_{\beta \lambda} \wedge h_{\gamma}^{\lambda}\right),
$$

которое позволяет использовать представление (3.17) для 2-формы, является следствием (3.21).

\section{4. КОМПЛЕКС ТОКОВ НА МАССОВОЙ ОБОЛОЧКЕ}

Изучим действие оператора $D(2.16)$ на массовой оболочке на дифференциальных формах, определенных в разделе 3 . Преимушество формулировки динамических уравнений в развернутой форме (2.14) состоит в том, что она выражает (внешнюю) пространственно-временную производную от $C$ через некоторые операторы, действуюшие во вспомогательном спинорном пространстве. В результате действие оператора $D$ на массовой оболочке сводится к некоторому отображению $\mathcal{D}$, действуюшему на коэффициенты в формулах (3.15)-(3.18).

Рассмотрим пример 0-форм. Используя правило Лейбница для $D^{L}$ и учитывая уравнения движения (2.14) и условие нулевого кручения $D^{L} h_{\alpha \alpha}=0(2.6)$, получаем

$$
\begin{aligned}
D \Phi_{0}(y, \psi \mid x)=\left(D^{L}-\lambda \psi h^{\alpha \beta} y_{\alpha} \frac{\partial}{\partial y^{\beta}}\right) \Phi_{0}(y, \psi \mid x)= \\
=\frac{i \lambda}{2} h^{\alpha \alpha} \frac{1}{(2 \pi)^{2}} \oint \frac{d z}{z} \oint \frac{d \bar{z}}{\bar{z}} \oint \frac{d \tau}{\tau^{2}} \int d^{2} q d^{2} \bar{q} \exp \left\{-\frac{1}{2 \tau}\left(q_{\gamma} \bar{q}^{\gamma}\right)\right\} E_{0}(z, \bar{z}, \tau) \times \\
\quad \times\left[4 \frac{\partial}{\partial q^{\alpha}} \frac{\partial}{\partial \bar{q}^{\alpha}}-q_{\alpha} \bar{q}_{\alpha}+i y_{\alpha}\left(\bar{z} q_{\alpha}-z \bar{q}_{\alpha}\right)-z \bar{z} y_{\alpha} y_{\alpha}-2 y_{\alpha}\left(z \frac{\partial}{\partial q^{\alpha}}-\bar{z} \frac{\partial}{\partial \bar{q}^{\alpha}}\right)\right] \times \\
\quad \times\left\{C\left[\frac{1}{2}(q+\bar{q})-\frac{1}{2 i}(z-\bar{z}) y, \psi \mid x\right] C^{\prime}\left[\frac{1}{2 i}(q-\bar{q})+\frac{1}{2}(z+\bar{z}) y, \psi \mid x\right]\right\} . \quad(4.1)
\end{aligned}
$$

Вьполняя интегрирование по частям по $q$ и $\bar{q}$, приходим к 1-форме $\widehat{\Phi}_{1}=D \Phi_{0}$ с коэффициентами $R_{1}^{\mathcal{D}}\left(E_{0}\right), W_{1}^{\mathcal{D}}\left(E_{0}\right), \bar{W}_{1}^{\mathcal{D}}\left(E_{0}\right), Y_{1}^{\mathcal{D}}\left(E_{0}\right), \bar{Y}_{1}^{\mathcal{D}}\left(E_{0}\right)$ и $V_{1}^{\mathcal{D}}\left(E_{0}\right)$ вида

$$
\begin{aligned}
R_{1}^{\mathcal{D}}\left(E_{0}\right) & =-\frac{i \lambda}{2} E_{0}(z, \bar{z}, \tau), \\
W_{1}^{\mathcal{D}}\left(E_{0}\right) & =i \lambda(1-i \tau) E_{0}(z, \bar{z}, \tau), \\
\bar{W}_{1}^{\mathcal{D}}\left(E_{0}\right) & =i \lambda(1+i \tau) E_{0}(z, \bar{z}, \tau), \\
V_{1}^{\mathcal{D}}\left(E_{0}\right) & =-i \lambda\left(1+\tau^{2}\right) E_{0}(z, \bar{z}, \tau), \\
Y_{1}^{\mathcal{D}}\left(E_{0}\right) & =\bar{Y}_{1}^{\mathcal{D}}\left(E_{0}\right)=0 .
\end{aligned}
$$

Аналогично выводится отображение $\mathcal{D}: \Phi_{i}(y) \rightarrow \widehat{\Phi}_{i+1}(y)=\left.D \Phi_{i}(y)\right|_{\text {on-shell }}, i=1,2$, на коэффишиентах дифференциальных форм (3.16)-(3.18),

$$
\mathcal{D}\left\{R_{1}, W_{1}, \ldots\right\}=\left\{R_{2}^{\mathcal{D}}, W_{2}^{\mathcal{D}}, \ldots\right\}, \quad \mathcal{D}\left\{R_{2}, W_{2}, \ldots\right\}=E_{3}^{\mathcal{D}},
$$


где

$$
\begin{aligned}
& R_{2}^{\mathcal{D}}=-(1-i \tau) n R_{1}-(1+i \tau) \bar{n} R_{1}+2 R_{1}+\frac{i}{4}(1+i \tau) \partial_{\tau} W_{1}-\frac{i}{4}(1-i \tau) \partial_{\tau} \bar{W}_{1}+ \\
& +\frac{1}{4}\left(W_{1}+\bar{W}_{1}\right)-\frac{1}{2}\left(n W_{1}+\bar{n} \bar{W}_{1}\right), \\
& W_{2}^{\mathcal{D}}=-\frac{i}{2} \partial_{\tau}\left[\left(1+\tau^{2}\right) W_{1}\right]+\frac{3}{2}(1+i \tau) W_{1}+2\left(1+\tau^{2}\right) \bar{n} R_{1}+\frac{1}{2}(1-i \tau) \bar{n} \bar{W}_{1}+ \\
& +(1-2 n) Y_{1}-\frac{1}{2}(1+i \tau)(\bar{n}-1) W_{1}+i(1+i \tau) \partial_{\tau} Y_{1}+ \\
& +\left(\frac{3}{2}-\bar{n}\right) V_{1}-\frac{i}{2}(1-i \tau) \partial_{\tau} V_{1} \\
& \bar{W}_{2}^{\mathcal{D}}=\frac{i}{2} \partial_{\tau}\left[\left(1+\tau^{2}\right) \bar{W}_{1}\right]+\frac{3}{2}(1-i \tau) \bar{W}_{1}+2\left(1+\tau^{2}\right) n R_{1}+\frac{1}{2}(1+i \tau) n W_{1}+ \\
& +(1-2 \bar{n}) \bar{Y}_{1}-\frac{1}{2}(1-i \tau)(n-1) \bar{W}_{1}-i(1-i \tau) \partial_{\tau} \bar{Y}_{1}+ \\
& +\left(\frac{3}{2}-n\right) V_{1}+\frac{i}{2}(1+i \tau) \partial_{\tau} V_{1} \\
& V_{2}^{\mathcal{D}}=\frac{1}{2}\left(1+\tau^{2}\right)\left(n W_{1}+\bar{n} \bar{W}_{1}\right)+\frac{1}{2}(1+i \tau)(\bar{n}-1) V_{1}+\frac{1}{2}(1-i \tau)(n-1) V_{1}+V_{1}+ \\
& +(1+i \tau) n Y_{1}+(1-i \tau) \bar{n} \bar{Y}_{1}, \\
& Y_{2}^{\mathcal{D}}=\frac{1}{2}\left(1+\tau^{2}\right)(\bar{n}-1) W_{1}-i\left(1+\tau^{2}\right) \partial_{\tau} Y_{1}+(1+i \tau) Y_{1}+(1-i \tau) n Y_{1}+ \\
& +\frac{1}{2}(1-i \tau)(\bar{n}-1) V_{1} \\
& \bar{Y}_{2}^{\mathcal{D}}=\frac{1}{2}\left(1+\tau^{2}\right)(n-1) \bar{W}_{1}+i\left(1+\tau^{2}\right) \partial_{\tau} \bar{Y}_{1}+(1-i \tau) \bar{Y}_{1}+(1+i \tau) \bar{n} \bar{Y}_{1}+ \\
& +\frac{1}{2}(1+i \tau)(n-1) V_{1}, \\
& E_{3}^{\mathcal{D}}=4 i n \bar{n}\left(1+\tau^{2}\right) R_{2}+i n \bar{n}\left(W_{2}+\bar{W}_{2}\right)-3 \tau\left(n W_{2}-\bar{n} \bar{W}_{2}\right)-\tau n \bar{n}\left(W_{2}-\bar{W}_{2}\right)+ \\
& +\partial_{\tau}\left[\left(1+\tau^{2}\right)\left(n W_{2}-\bar{n} \bar{W}_{2}\right)\right]+2 \partial_{\tau}\left(n Y_{2}-\bar{n} \bar{Y}_{2}\right)-(n-\bar{n}) \partial_{\tau} V_{2}+ \\
& +2 i(n \bar{n}-n-\bar{n}+1) V_{2}+i(n+\bar{n}-2) \tau \partial_{\tau} V_{2}+i\left(1+\tau^{2}\right) \partial_{\tau} \partial_{\tau} V_{2}- \\
& -3 i\left(n W_{2}+\bar{n} \bar{W}_{2}\right)+(i+\tau) n(n+1) W_{2}+(i-\tau) \bar{n}(\bar{n}+1) \bar{W}_{2}-4 i\left(n Y_{2}+\bar{n} \bar{Y}_{2}\right)+ \\
& +2 i \tau \partial_{\tau}\left(n Y_{2}+\bar{n} \bar{Y}_{2}\right)+2 i\left[n(n+1) Y_{2}+\bar{n}(\bar{n}+1) \bar{Y}_{2}\right] \text {. }
\end{aligned}
$$

Как и ожидалось, $\mathcal{D}^{2}=0$, и, следовательно, отображение $\mathcal{D}$ задает комплекс $(T, \mathcal{D})$, где

$$
T=\bigoplus_{i=0,1,2,3} T_{i}, \quad T_{0,3}=\left\{E_{0,3}\right\}, \quad T_{1,2}=\left\{R_{1,2}, W_{1,2}, \bar{W}_{1,2}, V_{1,2}, Y_{1,2}, \bar{Y}_{1,2}\right\}
$$

Переформулировка задачи в терминах $(T, \mathcal{D})$ эффективно учитывает тот факт, что поля лежат на массовой оболочке. Мы отождествляем когомологии токов с когомологиями оператора $\mathcal{D}$, действуюшего на пространстве $T$ (4.11).

Замечательным свойством отображения $\mathcal{D}$ является то, что оно содержит $z, \bar{z}, \partial / \partial z$ и $\partial / \partial \bar{z}$ только в виде комбинаций $n$ и $\bar{n}(3.19)$, что означает разделение переменных: дифференциал $\mathcal{D}$ оставляет инвариантными собственные подпространства операторов $n$ и $\bar{n}$. 
В результате функции $R_{1,2}, W_{1,2}, \ldots$ с одинаковыми значениями $n$ и $\bar{n}$ можно рассматривать независимо. В действительности это является главной причиной использования конкретного представления (3.15)-(3.18). Ясно, что это свойство значительно упрощает изучение когомологий токов, сводя его к анализу функций единственной переменной $\tau$ с двумя целыми параметрами $n$ и $\bar{n}$. Факт сушествования такого разделения переменных является следствием формы уравнений полей материи (2.14).

Как и ожидалось, система (4.4)-(4.9) совместна с преобразованиями Фирца (3.24)-(3.29), а именно любое преобразование Фирца для величин $R_{1}, W_{1}, \ldots$ приводит к преобразованию Фирца величин $R_{2}^{\mathcal{D}}, W_{2}^{\mathcal{D}}, \ldots$ с параметрами

$$
\begin{aligned}
\chi_{2}\left(\chi_{1}, \xi_{1}, \bar{\xi}_{1}\right)= & -\frac{1}{2}[(n+\bar{n})-i \tau(n-\bar{n})-4] \chi_{1}+ \\
& +\frac{1}{4}\left[i \partial_{\tau}\left(\xi_{1}-\bar{\xi}_{1}\right)+\left(1-\tau \partial_{\tau}\right)\left(\xi_{1}+\bar{\xi}_{1}\right)-2\left(n \xi_{1}+\bar{n} \bar{\xi}_{1}\right)\right], \\
\xi_{2}\left(\chi_{1}, \xi_{1}, \bar{\xi}_{1}\right)= & \left(1+\tau^{2}\right) \bar{n} \chi_{1}+\frac{1}{2}(1-i \tau) \bar{n} \bar{\xi}_{1}+ \\
& +\frac{1}{2}\left[-i\left(1+\tau^{2}\right) \partial_{\tau} \xi_{1}+(n+2) \xi_{1}-i \tau(n-2) \xi_{1}\right] \\
\bar{\xi}_{2}\left(\chi_{1}, \xi_{1}, \bar{\xi}_{1}\right)= & \left(1+\tau^{2}\right) n \chi_{1}+\frac{1}{2}(1+i \tau) n \xi_{1}+ \\
& +\frac{1}{2}\left[i\left(1+\tau^{2}\right) \partial_{\tau} \bar{\xi}_{1}+(\bar{n}+2) \bar{\xi}_{1}+i \tau(\bar{n}-2) \bar{\xi}_{1}\right]
\end{aligned}
$$

и никакое преобразование Фирца величин $R_{2}, W_{2}, \ldots$ не влияет на параметр $E_{3}^{\mathcal{D}}(4.10)$.

Конечно, формулы (4.4)-(4.9) совместны с произволом в добавлении тривиальных слагаемых (3.20) к величинам $R_{1}, W_{1}, \ldots$ в том смысле, что эти преобразования ведут к аналогичным преобразованиям величин $R_{2}^{\mathcal{D}}, W_{2}^{\mathcal{D}}, \ldots$, которые не влияют на 2-форму $\widehat{\Phi}_{2}(y)$.

\section{5. КОГОМолоГИИ ТОКОВ}

Следуя [13], мы изучим токи, которые содержат минимальное возможное число пространственно-временных производных для данного спина $s$. Согласно (2.12) в этом случае число свернутых индексов $\beta$ в 1-форме (3.1) должно равняться нулю. Поскольку число сверток равно - $\left(n_{\tau}+1\right)$ (см. раздел 3$)$, мы рассмотрим 2-формы $\Phi_{2}^{n, \bar{n}}$ с $n_{\tau}=-1$, полагая в (3.17)

$$
\begin{aligned}
& R_{2}=\alpha_{R}(n, \bar{n}) z^{n} \bar{z}^{\bar{n}} \tau^{-1}, \quad W_{2}=\alpha_{W}(n, \bar{n}) z^{n} \bar{z}^{\bar{n}} \tau^{-1}, \bar{W}_{2}=\alpha_{\bar{W}}(n, \bar{n}) z^{n} \bar{z}^{\bar{n}} \tau^{-1}, \\
& Y_{2}=\alpha_{Y}(n, \bar{n}) z^{n} \bar{z}^{\bar{n}} \tau^{-1}, \quad \bar{Y}_{2}=\alpha_{\bar{Y}}(n, \bar{n}) z^{n} \bar{z}^{\bar{n}} \tau^{-1}, \quad V_{2}=\alpha_{V}(n, \bar{n}) z^{n} \bar{z}^{\bar{n}} \tau^{-1}
\end{aligned}
$$

с некоторыми постоянными параметрами $\alpha_{R}(n, \bar{n}), \alpha_{W}(n, \bar{n}), \ldots$, пропорциональными $\lambda^{[s]}$, где $s=1-(n+\bar{n}) / 2$. Условие сохранения означает, что форма $\Phi_{2}^{n, \bar{n}}$ должна быть $\mathcal{D}$-замкнутой. Требование, чтобы $E_{3}^{\mathcal{D}}=0$ с точностью до слагаемых, которые не дают вклада в (3.18), налагает следующие условия на параметры в (5.1):

$$
\begin{gathered}
4 n \bar{n} \alpha_{R}+(n+\bar{n}-2)\left(n \alpha_{W}+\bar{n} \alpha_{\bar{W}}\right)+2 n(n-2) \alpha_{Y}+2 \bar{n}(\bar{n}-2) \alpha_{\bar{Y}}=0, \\
n \alpha_{W}-\bar{n} \alpha_{\bar{W}}+2\left(n \alpha_{Y}-\bar{n} \alpha_{\bar{Y}}\right)=0, \\
\alpha_{V}=0,
\end{gathered}
$$


при $n \neq 1, \bar{n} \neq 1$. При $n=1$ или $\bar{n}=1$ форма $\Phi_{2}^{n,}, \bar{n}$ замкнута вследствие условий на $E_{3}^{\mathcal{D}}$ (см. таблицу).

Наша задача состоит в ответе на вопрос, существуют ли такие параметры $R_{1}, W_{1}, \ldots$, что $R_{2}^{\mathcal{D}}, W_{2}^{\mathcal{D}}, \ldots$ имеют вид (5.1). Для этого необходимо решить систему $(4.4)-(4.9)$ в терминах формальных рядов

$$
f(\tau)=\sum_{k=-\infty}^{p<\infty} f_{k} \tau^{k} .
$$

Согласно тождествам (3.21) имеется произвол, связанный с преобразованиями Фирца $(3.24)-(3.29)$ для $\Phi_{1,2}$. Также можно использовать неоднозначность в “точных сдвигах" величин $R_{1}, W_{1}, \ldots$ на любые $R_{1}^{\mathcal{D}}, W_{1}^{\mathcal{D}}, \ldots(4.2)$, которые не влияют на $\Phi_{2}$, поскольку $\mathcal{D}^{2}=0$. "Точные сдвиги" вместе с преобразованиями Фирца для $\Phi_{1}$ образуют следуюшие преобразования эквивалентности:

$$
\begin{aligned}
\delta R_{1} & =-\partial_{\tau} \chi_{1}+\varepsilon \\
\delta W_{1} & =-\partial_{\tau} \xi_{1}+2 i \bar{n} \chi_{1}-2(1-i \tau) \varepsilon \\
\delta \bar{W}_{1} & =-\partial_{\tau} \bar{\xi}_{1}-2 i n \chi_{1}-2(1+i \tau) \varepsilon \\
\delta V_{1} & =2\left(1+\tau^{2}\right) \varepsilon-i\left(n \xi_{1}-\bar{n} \bar{\xi}_{1}\right), \\
\delta Y_{1} & =i(\bar{n}-1) \xi_{1} \\
\delta \bar{Y}_{1} & =-i(n-1) \bar{\xi}_{1}
\end{aligned}
$$

где в соответствии с $(4.2) \varepsilon(z, \bar{z}, \tau)=-(i \lambda / 2) E_{0}(z, \bar{z}, \tau)$.

Для данного спина $s$ мы рассмотрим отдельно два случая:

1) $n=1, \bar{n}=1-2 s$ или $\bar{n}=1, n=1-2 s$;

2) $n<1$ и $\bar{n}<1$.

Как показано ниже, случай 1 соответствует физическим сохраняющимся токам, в то время как случай 2 описывает все возможные "улучшения".

Начнем со случая 1 , полагая для определенности $\bar{n}=1$. Случай $n=1$ может быть рассмотрен аналогично. Согласно таблице единственным параметром, дающим ненулевой вклад в $\Phi_{2}^{n, 1}(y)$, является $Y_{2}$. Очевидно, что 2-форма с $\bar{n}=1$ инвариантна относительно преобразований (3.28). Единственным нетривиальным уравнением является уравнение (4.8); с параметром $Y_{2}^{\mathcal{D}}$ в виде (5.1) оно может быть переписано следующим образом:

$$
\left(1+\tau^{2}\right) \frac{\partial}{\partial \tau} Y_{1}=-i(1+i \tau) Y_{1}+i(2 s-1)(1-i \tau) Y_{1}+i \alpha_{Y}(1-2 s, 1) \frac{z^{1-2 s} \bar{z}}{\tau}+P(\tau)
$$

где $P(\tau)$ - некоторый полином (3.20). Как показано в приложении А, обшее решение уравнения (5.12) есть

$$
\begin{aligned}
Y_{1}(z, \bar{z}, \tau)= & -\frac{i}{2} \alpha_{Y}(1-2 s, 1) z^{1-2 s} \bar{z}(1-i \tau)(1+i \tau)^{2 s-1} \ln \left(1+\tau^{-2}\right)+ \\
& +\sigma z^{1-2 s} \bar{z}(1-i \tau)(1+i \tau)^{2 s-1} \ln \left(\frac{1+i \tau^{-1}}{1-i \tau^{-1}}\right)+Q(\tau)
\end{aligned}
$$

где $\sigma$ - произвольная константа, а $Q(\tau)$ - некоторый несущественный полином вида (3.20). Логарифмы рассматриваются как ряды по $\tau^{-1}$. 
При любом $\sigma$ решение (5.13) есть бесконечный ряд по $\tau^{-1}$, и тем самым оно соответствует некоторой псевдолокальной 1-форме. Следовательно, 2-формы $\Phi_{2}^{s}(y \mid x)$, построенные с помощью полиномов $Y_{2}$ при $\bar{n}=1$ и полиномов $\bar{Y}_{2}$ при $n=1$, являются $\mathcal{D}$-замкнутыми, но не могут быть представлены в виде $D \Phi_{1}^{S}(y \mid x)$ с пространственно-локальной 1-формой $\Phi_{1}^{s}(y \mid x)$. Следовательно, мы заключаем, что 2-форма $\Phi_{2}^{s}(y)$ описывает физические сохраняюшиеся токи спина $s$. Токи (2.18)-(2.20) воспроизводятся с помощью $Y_{2}(5.1)$ при

$$
\alpha_{Y}(1-2 s, 1)=2^{2 s-1}(\lambda \psi)^{[s]} .
$$

Формула (5.13) решает задачу о переформулировке физических токов в виде псевдолокально-точных 2-форм.

Заметим, что 1- и 2-формы (3.16), (3.17) имеют следуюшую дискретную симметрию, переставляющую $C$ и $C^{\prime}$ :

$$
\Phi\left[C(y), C^{\prime}(y) ; y\right]=(-)^{\pi(C) \pi\left(C^{\prime}\right)+1} \Phi^{\prime}\left[C^{\prime}(i y), C(i y) ;-y\right],
$$

где $\Phi^{\prime}(y)$ задается параметрами

$$
\left(R^{\prime}, W^{\prime}, \bar{W}^{\prime}, V^{\prime}, Y^{\prime}, \bar{Y}^{\prime}\right)(z, \bar{z}, \tau)=(R,-\bar{W},-W, V, \bar{Y}, Y)(-\bar{z}, z, \tau) .
$$

Эквивалентность токов, порождаемых параметрами $Y_{2}(n=1-2 s, \bar{n}=1)$ и $\bar{Y}_{2}(n=1, \bar{n}=1-2 s)$, устанавливается перестановкой $C \leftrightarrow C^{\prime}$.

Заметим, что решение (5.13) не единственно, а содержит произвольный параметр $\sigma$. Поскольку преобразования (5.6)-(5.11) тривиальны для $Y_{1}$ при $\bar{n}=1$, этот однопараметрический произвол не может быть скомпенсирован таким образом. Это означает, что мы нашли псевдолокальную 1-форму, которая $\mathcal{D}$-замкнута, но не является $\mathcal{D}$-точной, т.е. группа когомологий $H^{1}(T, \mathcal{D})$ нетривиальна. Физический смысл этого явления нам до конца не ясен. Этот результат находится, однако, в согласии с однопараметрической неоднозначностью, найденной в работе [10] для случая спина 2.

Рассмотрим теперь случай $n<1, \bar{n}<1$. Подставляя (5.1) в систему (4.4)-(4.9), мы показываем в приложении Б, что если выполнены условия (5.2)-(5.4), обеспечиваюшие $\mathcal{D}$-замкнутость формы $\Phi_{2}$, то с точностью до калибровочных преобразований (5.6)-(5.11) общее решение системы есть

$$
\begin{aligned}
R_{1}(z, \bar{z}, \tau)= & \frac{z^{n} \bar{z} \bar{n}}{4 \tau n \bar{n}}\left[n \alpha_{W}(n, \bar{n})+\bar{n} \alpha_{\bar{W}}(n, \bar{n})-\right. \\
& \left.-\frac{2 n}{\bar{n}-1} \alpha_{Y}(n, \bar{n})-\frac{2 \bar{n}}{n-1} \alpha_{\bar{Y}}(n, \bar{n})\right] \quad(n<0, \quad \bar{n}<0), \\
W_{1}(z, \bar{z}, \tau)= & \frac{\alpha_{Y}(n, \bar{n})}{\bar{n}-1} \frac{z^{n} \bar{z}^{\bar{n}}}{\tau}+ \\
& +\sigma(n, \bar{n}) z^{n} \bar{z} \bar{n}\left[-\frac{i \tau^{-1}}{1-i \tau^{-1}}+\frac{\bar{n}}{2} \ln \left(\frac{1+i \tau^{-1}}{1-i \tau^{-1}}\right)\right] \\
\bar{W}_{1}(z, \bar{z}, \tau)= & \frac{\alpha_{\bar{Y}}(n, \bar{n})}{n-1} \frac{z^{n} \bar{z}^{\bar{n}}}{\tau}+ \\
& +\sigma(n, \bar{n}) z^{n} \bar{z} \bar{n}\left[\frac{i \tau^{-1}}{1+i \tau^{-1}}-\frac{n}{2} \ln \left(\frac{1+i \tau^{-1}}{1-i \tau^{-1}}\right)\right] \\
& V_{1}(z, \bar{z}, \tau)=Y_{1}(z, \bar{z}, \tau)=\bar{Y}_{1}(z, \bar{z}, \tau)=0
\end{aligned}
$$


где $\sigma(n, \bar{n})$ - свободные параметры. При $n=\bar{n}=0$ необходимо положить $\alpha_{V}(0,0)=0$, и решение оказывается чисто калибровочным.

Мы видим, что при $\sigma(n, \bar{n})=0$ 1-форма $\Phi_{1}^{n, \bar{n}}(y)$ приводит к пространственно-локальному выражению, так как $R_{1}, W_{1}$ и $\bar{W}_{1}(5.17)-(5.19)$ линейны по $\tau^{-1}$. Следовательно, 2-форма $\widehat{\Phi}_{2}^{n, \bar{n}}(y \mid x)=D \Phi_{1}^{n, \bar{n}}(y \mid x)$ с локальной 1-формой $\Phi_{1}^{n, \bar{n}}(y \mid x)$, и поэтому она является “улучшением" 2-формы физического тока $J\left(C^{2}\right)$, стоящей в правой части (2.15). Соответствуюшие члены могут быть скомпенсированы локальным переопределением калибровочных полей (высших спинов).

Вновь произвол, связанный с параметрами $\sigma(n, \bar{n})$, является проявлением нетривиальной когомологии. В результате мы заключаем, что группа $H^{1}\left(T^{n, \bar{n}}, \mathcal{D}\right)$ одномерна в каждом секторе $(n, \bar{n})$. Следовательно, для данного спина $s=1-(n+\bar{n}) / 2$ $\operatorname{dim} H^{1}\left(T^{s}, \mathcal{D}\right)=2 s+1$, что, конечно, совпадает с размерностью представления спина $s$ трехмерной алгебры Лоренца $o(2,1)$.

Таким образом, мы показали, что все локальные $\mathcal{D}$-замкнутые формы $\Phi_{2}^{n, \bar{n}}(y)$ с $n_{\tau}=-1$ являются $\mathcal{D}$-точными в классе псевдолокальных разложений. Физические сохраняющиеся токи описываются формами $\Phi_{2}^{1,1-2 s}(y)$ или, что эквивалентно, формами $\Phi_{2}^{1-2 s, 1}(y) . \quad \mathcal{D}$-замкнутые формы $\Phi_{2}^{n, \bar{n}}(y)$ с $n, \bar{n} \leqslant 0$ локально $\mathcal{D}$-точны и, следовательно, описывают всевозможные “улучшения".

\section{6. ЗАВИСИМОСТЬ ОТ $\lambda$}

Обшие $p$-формы, задаваемые формулами (3.15)-(3.18), зависят от $\lambda$ через разложения (2.13). Такая формулировка с зависящими от $\lambda$ функциями $C(y)$ и $C^{\prime}(y)$ была удобна для изучения когомологий, так как она позволяла использовать не зависящие от $\lambda$ формулы для отображения $\mathcal{D}(4.2),(4.4)-(4.10)$ и решений (5.13), (5.17)-(5.19). Однако в плоском пределе $\lambda \rightarrow 0$ разложения $C(y)$ становятся бессмысленными, и для исследования того, что происходит в этом случае, необходимо использовать производяшие функции $\widetilde{C}(y)(2.13)$. В этих переменных решения (5.13), (5.17)-(5.19) приобретают явную зависимость от $\lambda$. Например, вводя формы

$$
\widetilde{\Phi}_{1,2}\left(\widetilde{C}, \widetilde{C}^{\prime} ; y\right)=\lambda^{-2-\frac{1}{2} \pi(C)-\frac{1}{2} \pi\left(C^{\prime}\right)} \Phi_{1,2}\left(C, C^{\prime} ; \sqrt{\lambda} y\right),
$$

мы получаем

$$
\begin{aligned}
& \widetilde{\Phi}_{1}\left(\widetilde{C}, \widetilde{C}^{\prime} ; y\right)=h_{\alpha \alpha} \frac{1}{(2 \pi)^{2}} \oint d z \oint d \bar{z} \oint \tau^{-2} d \tau \int d^{2} q d^{2} \bar{q} \exp \left\{-\frac{1}{2 \tau}\left(q_{\gamma} \bar{q}^{\gamma}\right)\right\} \times \\
& \quad \times \widetilde{C}\left[\frac{1}{2}(q+\bar{q})-\frac{1}{2 i}(z-\bar{z}) y, \psi \mid x\right] \widetilde{C}^{\prime}\left[\frac{1}{2 i}(q-\bar{q})+\frac{1}{2}(z+\bar{z}) y, \psi \mid x\right] \times \\
& \quad \times\left\{\widetilde{R}_{1}(z, \bar{z}, \tau) y^{\alpha} y^{\alpha}+\frac{1}{2 \tau \bar{z}} \widetilde{W}_{1}(z, \bar{z}, \tau) y^{\alpha} \bar{q}^{\alpha}+\frac{1}{2 \tau z} \widetilde{W}_{1}(z, \bar{z}, \tau) y^{\alpha} q^{\alpha}+\right. \\
& \left.\quad+\frac{1}{2 \tau^{2} \bar{z}^{2}} \widetilde{Y}_{1}(z, \bar{z}, \tau) \bar{q}^{\alpha} \bar{q}^{\alpha}+\frac{1}{2 \tau^{2} z^{2}} \widetilde{\bar{Y}}_{1}(z, \bar{z}, \tau) q^{\alpha} q^{\alpha}+\frac{1}{2 \tau^{2} z \bar{z}} \widetilde{V}_{1}(z, \bar{z}, \tau) q^{\alpha} \bar{q}^{\alpha}\right\},
\end{aligned}
$$


где

$$
\begin{aligned}
\widetilde{R}_{1}(z, \bar{z}, \tau) & =R_{1}(z, \bar{z}, \lambda \tau), \\
\widetilde{W}_{1}(z, \bar{z}, \tau) & =\lambda^{-1} W_{1}(z, \bar{z}, \lambda \tau), \\
\widetilde{W}_{1}(z, \bar{z}, \tau) & =\lambda^{-1} \bar{W}_{1}(z, \bar{z}, \lambda \tau), \\
\widetilde{Y}_{1}(z, \bar{z}, \tau) & =\lambda^{-2} Y_{1}(z, \bar{z}, \lambda \tau), \\
\widetilde{\bar{Y}}_{1}(z, \bar{z}, \tau) & =\lambda^{-2} \bar{Y}_{1}(z, \bar{z}, \lambda \tau), \\
\widetilde{V}_{1}(z, \bar{z}, \tau) & =\lambda^{-2} V_{1}(z, \bar{z}, \lambda \tau) .
\end{aligned}
$$

Следовательно, в терминах $\widetilde{R}, \widetilde{W}, \ldots$ наши решения содержат отрицательную степень $\lambda$ при каждой степени $\tau^{-1}$. Эквивалентно каждое пространственно-временное дифференцирование содержит фактор $\lambda^{-1}$. Таким образом, представление 2-форм физических токов $\Phi_{2}^{s}(y)$ в виде некоторых дифференциалов $D \Phi_{1}^{s}(y)$, а также все элементы группы когомологий $H^{1}(T, \mathcal{D})$ становятся бессмысленными в плоском пределе $\lambda \rightarrow 0$. В переменных $\widetilde{C}(y)$ выражения $(5.13),(5.18)$ и (5.19) содержат комбинации $\left(1 \pm i \lambda^{-1} \tau^{-1}\right)^{-1}$, разлагаемые по степеням $\tau^{-1}$. Интересно, что эти выражения, рассматриваемые как аналитические функции, имеют радиус сходимости, равный $\lambda$, и когда радиус $\mathrm{AdS} \lambda^{-1}$ стремится к бесконечности, их радиус сходимости стремится к нулю.

\section{ЗАКЛЮЧЕНИЕ}

В данной работе мы построили из безмассовых скалярных и спинорных полей локальные сохраняющиеся токи произвольного спина в $\mathrm{AdS}_{3}$ и показали, что они могут рассматриваться как "улучшения" в классе бесконечных разложений по старшим производным. Другими словами, показано, что в этом классе 2-формы $J$, дуальные физическим сохраняющимся токам, являются точными, т.е. $J=D U$. 1-формы $U$ построены явно, что позволяет выписать нелокальные переопределения полей, компенсирующие материальные источники в уравнениях движения для калибровочных полей Черна-Саймонса всех спинов. Коэффициенты в разложениях 1-форм $U$ по производным полей материи содержат отрицательные степени космологической постоянной (т.е. положительные степени радиуса $\mathrm{AdS}$ ) и, следовательно, не допускают плоского предела. Сушествование таких 1-форм $U$ может быть связано с голографичностью соответствия $\mathrm{AdS} / \mathrm{CFT}$ (между теориями в пространстве AdS и конформными теориями на границе пространства AdS) $[2,3]$ и указывает на то, что взаимодействия с локальными токами в пространстве AdS в определенном смысле тривиальны и могут быть скомпенсированы переопределением полей с точностью до некоторых поверхностных членов.

Отметим, что наш анализ с двумя независимыми полями материи $C$ и $C^{\prime}$ в $(3.1)$ тривиально обобшается на случай с полями материи, лежащими в нетривиальном представлении группы Янга-Миллса (спин 1) в расширенных системах, рассмотренных в рабо$\operatorname{Tax}[9,10]$.

Для систематического изучения задачи о классификации токов всех спинов мы предложили формализм производящих функций, удобный для описания дифференциальных форм, билинейных по безмассовым скалярным и спинорным полям. Он основан на развернутой формулировке динамических уравнений в виде некоторых условий ковариантного постоянства [11] и позволяет перефформулировать задачу на языке когомологий не- 
которого дифференциала $\mathcal{D}$, действующего в определенных вспомогательных пространствах, содержаших полную информацию о полях материи на массовой оболочке.

Нашим основным результатом является установление того факта, что локальные сохраняюшиеся (т.е. $\mathcal{D}$-замкнутые) 2-формы токов, которые относятся к нетривиальным когомологиям в классе локальных разложений, являются $\mathcal{D}$-точными в классе псевдолокальных разложений (т.е. бесконечных рядов по высшим производным). Довольно интересным побочным результатом является нахождение групшы когомологий $H^{1}(T, \mathcal{D})$, которая указывает на неоднозначность решения для $U$, уже отмеченную в работе [10] в случае спина 2. Интересно было бы также найти теоретико-групповое объяснение того факта, что размерность группы $H^{1}\left(T^{s}, \mathcal{D}\right)$ в секторе спина $s$ совпадает с размерностью представления спина $s$ трехмерной алгебры Лоренца $o(2,1)$, и изучить $H^{n}\left(T^{s}, \mathcal{D}\right)$ при $n \neq 1$. Поскольку в работе [10] было показано, что существует псевдолокальная замена переменных, сводяшая полные нелинейные уравнения движения к свободной системе, мы ожидаем, что $H^{2}(T, \mathcal{D})=0$; тем не менее представляется интересным проанализировать эту задачу независимо. В частности, это могло бы пролить свет на вопрос об адекватном определении локальных функционалов в пространстве AdS как некоторого подпространства класса формальных псевдолокальных разложений.

Благодарности. Данная работа частично поддержана грантами INTAS № 96-0538 и РФФИ № 96-15-96463 и № 99-02-17916. С. П. благодарит за частичную поддержку Фонд стипендии Ландау (Forschungszentrum Jülich).

\section{Решение при $\bar{n}=1$}

\section{ПРИЛОЖЕНИЕ А}

Рассмотрим следующее дифференциальное уравнение, которое связано с уравнением (5.12) заменой $x=i \tau, k=2 s-1, \alpha=i \alpha_{Y}(n, \bar{n}) z^{1-2 s} \bar{z}$ и $Y=Y_{1}$ :

$$
\left(1-x^{2}\right) \frac{d}{d x} Y=-(1+x) Y+k(1-x) Y+\frac{\alpha}{x}+P(x),
$$

где $k$ и $\alpha$-некоторые константы, а $P(x)$ - произвольный полином. Уравнение (А.1) следует решать в терминах формальных рядов (5.5). Существенная часть $Y(x)$ содержит отрицательные степени $x$, т.е. $Y(x)$ определено с точностью до произвольных полиномов.

Переписывая уравнение (А.1) в виде

$$
(1+x)^{k+1}(1-x)^{2} \frac{d}{d x}\left[(1+x)^{-k}(1-x)^{-1} Y\right]=\frac{\alpha}{x}+P(x),
$$

находим решение в виде

$$
\begin{aligned}
Y & =(1+x)^{k}(1-x) \int_{c}^{x} d t(1+t)^{-k-1}(1-t)^{-2}\left(\frac{\alpha}{t}+P(t)\right)= \\
& =(1+x)^{k}(1-x) \int_{c}^{x} d t\left[\frac{\alpha}{t}+(1+t)^{-k-1}(1-t)^{-2} P^{\prime}(t)\right]
\end{aligned}
$$

с полиномом $P^{\prime}(t)=P(t)-\alpha t^{-1}\left[(1+t)^{k+1}(1-t)^{2}-1\right]$. С помощью формулы

$$
\frac{2}{(1+t)(1-t)}=\frac{1}{(1-t)}+\frac{1}{(1+t)}
$$


находим, что $P^{\prime}(t)$ может давать неполиномиальный вклад в $Y$, только если простые полюсы по $(1 \pm t)$ содержатся под интегралом в (А.3). Эквивалентно можно положить

$$
P^{\prime}(t)=(1+t)^{k}(1-t)[\beta(1-t)+\gamma(1+t)]
$$

Таким образом, обшее решение уравнения (А.1) есть

$$
Y(x)=(1-x)(1+x)^{k}[\alpha \ln x+\beta \ln (1+x)+\gamma \ln (1-x)]+P(x),
$$

где $P(x)$ - произвольный полином. Ограничение на класс (5.5) налагает одно условие на параметры $\beta$ и $\gamma$, что дает окончательный ответ

$$
Y(x)=-\frac{\alpha}{2}(1-x)(1+x)^{k} \ln \left(1-x^{-2}\right)+\sigma(1-x)(1+x)^{k} \ln \left(\frac{1-x^{-1}}{1+x^{-1}}\right)
$$

с произвольной константой $\sigma$. Заметим, что логарифмы в (А.7) понимаются как ряды

$$
\ln \left(1+x^{-1}\right)=\sum_{m=1}^{\infty} \frac{(-)^{m-1}}{m} x^{-m}
$$

\section{Решение при $n<1, \bar{n}<1$}

ПРИЛОЖЕНИЕ Б

Рассмотрим сначала случай $n, \bar{n}<0$. Чтобы решить систему (4.4)-(4.9) с параметрами $R_{2}^{\mathcal{D}}, W_{2}^{\mathcal{D}}, \ldots$ вида $(5.1)$, наиболее удобно зафиксировать калибровку величин $R_{1}$, $W_{1}, \ldots$ в 1-форме $\Phi_{1}^{n, \bar{n}}(y)$ следуюшим образом:

$$
V_{1}=Y_{1}=\bar{Y}_{1}=0, \quad R_{1}=\frac{r}{\tau}
$$

с некоторым $r$, не зависяшим от $\tau$. Действительно, при $n \neq 1, \bar{n} \neq 1$ всегда можно добиться выполнения (Б.1), используя преобразования (5.6)-(5.11). Прежде всего, $Y_{1}$ и $\bar{Y}_{1}$ откалибровываются путем подходящего выбора параметров $\xi_{1}$ и $\bar{\xi}_{1}$. Выбирая параметр $\varepsilon(\tau)$ в $(5.9)$ в виде

$$
\varepsilon(\tau)=\frac{1}{1+\tau^{2}}\left[E\left(\tau^{-1}\right)+\varepsilon_{0} \tau\right]
$$

где $E\left(\tau^{-1}\right)$ - некоторый ряд по отрицательным степеням $\tau$, а $\varepsilon_{0}$ не зависит от $\tau$, можно откалибровать $V_{1}$ подходяшим выбором $E\left(\tau^{-1}\right)$. Параметр $\varepsilon_{0}$ остается произвольным, поскольку его вклад в $V_{1}$ пропорционален $\tau$, что эквивалентно нулю. Из (5.6) следует, что все члены в $R_{1}$ могут быть откалиброваны путем выбора некоторого $\chi(\tau)$, за исключением ведушего члена $\tau^{-1}$. Константу $r$ в члене $r \tau^{-1}$ в $R_{1}$ можно зафиксировать, используя произвол в выборе $\varepsilon_{0}$, поскольку соответствующая часть $\varepsilon(\tau)$,

$$
\varepsilon_{0} \frac{\tau}{1+\tau^{2}}=\varepsilon_{0} \sum_{k=0}^{\infty}(-)^{k} \tau^{-(2 k+1)},
$$

содержит член, пропорциональный $\tau^{-1}$. На данном этапе, однако, удобно рассматривать $r$ как произвольный параметр, который будет зафиксирован позже. Отметим, что 
фиксация $\varepsilon_{0}$ завершает фиксацию калибровки, связанной с преобразованиями $(5.6)-(5.11)$.

В результате система уравнений, следующая из (4.4)-(4.9), принимает вид

$$
\begin{aligned}
\alpha_{R}(n, \bar{n}) \frac{z^{n} \bar{z} \bar{n}}{\tau}= & -(n+\bar{n}-2) \frac{r}{\tau}+\frac{i}{4}(1+i \tau) \partial_{\tau} W_{1}-\frac{i}{4}(1-i \tau) \partial_{\tau} \bar{W}_{1}+ \\
& +\frac{1}{4}\left(W_{1}+\bar{W}_{1}\right)-\frac{1}{2}\left(n W_{1}+\bar{n} \bar{W}_{1}\right)-\partial_{\tau} \chi_{2}, \\
\alpha_{W}(n, \bar{n}) \frac{z^{n} \bar{z} \bar{n}}{\tau}= & -\frac{i}{2} \partial_{\tau}\left[\left(1+\tau^{2}\right) W_{1}\right]+\frac{3}{2}(1+i \tau) W_{1}+2 \bar{n} \frac{r}{\tau}+ \\
& +\frac{1}{2}(1-i \tau) \bar{n} \bar{W}_{1}-\frac{1}{2}(1+i \tau)(\bar{n}-1) W_{1}-\partial_{\tau} \xi_{2}+2 i \bar{n} \chi_{2}, \\
\alpha_{\bar{W}}(n, \bar{n}) \frac{z^{n} \bar{z} \bar{n}}{\tau}= & \frac{i}{2} \partial_{\tau}\left[\left(1+\tau^{2}\right) \bar{W}_{1}\right]+\frac{3}{2}(1-i \tau) \bar{W}_{1}+2 n \frac{r}{\tau}+ \\
& +\frac{1}{2}(1+i \tau) n W_{1}-\frac{1}{2}(1-i \tau)(n-1) \bar{W}_{1}-\partial_{\tau} \bar{\xi}_{2}-2 i n \chi_{2}, \\
\alpha_{V}(n, \bar{n}) \frac{z^{n} \bar{z} \bar{n}}{\tau}= & \frac{1}{2}\left(1+\tau^{2}\right)\left(n W_{1}+\bar{n} \bar{W}_{1}\right)-i\left(n \xi_{2}-\bar{n} \bar{\xi}_{2}\right), \\
\alpha_{Y}(n, \bar{n}) \frac{z^{n} \bar{z} \bar{n}}{\tau}= & \frac{1}{2}\left(1+\tau^{2}\right)(\bar{n}-1) W_{1}+i(\bar{n}-1) \xi_{2}, \\
\alpha_{\bar{Y}}(n, \bar{n}) \frac{z^{n} \bar{z} \bar{n}}{\tau}= & \frac{1}{2}\left(1+\tau^{2}\right)(n-1) \bar{W}_{1}-i(n-1) \bar{\xi}_{2},
\end{aligned}
$$

где все равенства рассматриваются с точностью до полиномов (3.20), а переменные $\chi_{2}, \xi_{2}$ и $\bar{\xi}_{2}$ учитывают произвол, связанный с преобразованиями Фирца величин $R_{2}^{\mathcal{D}}$, $W_{2}^{\mathcal{D}}, \ldots$.

Вводя новые переменные

$$
X=n W_{1}-\bar{n} \bar{W}_{1}, \quad X^{+}=n W_{1}+\bar{n} \bar{W}_{1}
$$

и выражая параметры Фирца $\chi_{2}, \xi_{2}$ и $\bar{\xi}_{2}$ через остальные переменные, сводим систему (Б.4)-(Б.9) к следующим уравнениям:

$$
\begin{gathered}
\left(1+\tau^{2}\right) X^{+}=\frac{A}{\tau}+P_{1}(\tau), \\
\left(1+\tau^{2}\right) \frac{\partial}{\partial \tau} X=-2 i X^{+}-\frac{i(4 n \bar{n} r-G)}{\tau}-\frac{B}{\tau^{2}}+P_{2}(\tau), \\
4 n \bar{n} \frac{\alpha_{R}}{\tau}+4 n \bar{n}(n+\bar{n}-2) \frac{r}{\tau}-[\tau(n-\bar{n})+i(n+\bar{n}-2)] \partial_{\tau} X+ \\
+[i(n-\bar{n})+\tau(n+\bar{n}-2)] \partial_{\tau} X^{+}+ \\
+2(n \bar{n}-1) X^{+}+\frac{i K}{\tau^{2}}+\frac{A-L}{\tau^{3}}=P_{3}(\tau),
\end{gathered}
$$


где

$$
\begin{aligned}
A & =\left(\frac{n}{\bar{n}-1} \alpha_{Y}+\frac{\bar{n}}{n-1} \alpha_{\bar{Y}}+\alpha_{V}\right) z^{n} \bar{z}^{\bar{n}}, \\
B & =\left(\frac{n}{\bar{n}-1} \alpha_{Y}-\frac{\bar{n}}{n-1} \alpha_{\bar{Y}}\right) z^{n} \bar{z}^{\bar{n}}, \\
G & =\left(n \alpha_{W}+\bar{n} \alpha_{\bar{W}}\right) z^{n} \bar{z} \bar{n} \\
K & =\left(n \alpha_{W}-\bar{n} \alpha_{\bar{W}}\right) z^{n} \bar{z}^{\bar{n}}, \\
L & =\left(\frac{n}{\bar{n}-1} \alpha_{Y}+\frac{\bar{n}}{n-1} \alpha_{\bar{Y}}-\alpha_{V}\right) z^{n} \bar{z}^{\bar{n}},
\end{aligned}
$$

а $P_{1}(\tau), P_{2}(\tau)$ и $P_{3}(\tau)$ - произвольные полиномы.

Используя тот факт, что любой полином $P(\tau)$ может быть переписан в виде

$$
P(\tau)=a+b \tau+\left(1+\tau^{2}\right) p(\tau),
$$

где $p(\tau)$ - некоторый другой полином, получаем обшее решение уравнения (Б.11):

$$
X^{+}(\tau)=\frac{A}{\tau}+\sigma_{1}^{+} z^{n} \bar{z}^{\bar{n}} \frac{1}{1+\tau^{2}}+\sigma_{2}^{+} z^{n} \bar{z}^{\bar{n}} \frac{\tau}{1+\tau^{2}}+p(\tau)
$$

где $\sigma_{1}^{+}$и $\sigma_{2}^{+}-$произвольные константы.

Подставляя (Б.16) в (Б.12) и разрешая последнее относительно $\partial_{\tau} X$ аналогично случаю (Б.11), получаем

$$
\begin{aligned}
\frac{\partial}{\partial \tau} X= & -\frac{i[4 n \bar{n} r-(G-2 A)]}{\tau\left(1+\tau^{2}\right)}-\frac{B}{\tau^{2}}-2 i \sigma_{1}^{+} z^{n} \bar{z}^{\bar{n}} \frac{1}{\left(1+\tau^{2}\right)^{2}}-2 i \sigma_{2}^{+} z^{n} \bar{z}^{\bar{n}} \frac{\tau}{\left(1+\tau^{2}\right)^{2}}+ \\
& +\sigma_{1} z^{n} \bar{z}^{\bar{n}} \frac{1}{1+\tau^{2}}+\sigma_{2} z^{n} \bar{z}^{\bar{n}} \frac{\tau}{1+\tau^{2}},
\end{aligned}
$$

где $\sigma_{1}$ и $\sigma_{2}-$ произвольные константы. Теперь удобно зафиксировать

$$
r=\frac{G-2 A}{4 n \bar{n}}
$$

Также необходимо положить $\sigma_{2}=0$ в (Б.17), поскольку член $\tau\left(1+\tau^{2}\right)^{-1}$ не интегрируется в виде (5.5). В результате мы приходим к дифференциальному уравнению

$$
\frac{\partial}{\partial \tau} X=-\frac{B}{\tau^{2}}-2 i \sigma_{1}^{+} z^{n} \bar{z}^{\bar{n}} \frac{1}{\left(1+\tau^{2}\right)^{2}}-2 i \sigma_{2}^{+} z^{n} \bar{z}^{\bar{n}} \frac{\tau}{\left(1+\tau^{2}\right)^{2}}+\sigma_{1} z^{n} \bar{z}^{\bar{n}} \frac{1}{1+\tau^{2}},
$$

и, следовательно, с точностью до полиномов

$$
\begin{aligned}
X(\tau)= & \frac{B}{\tau}-i \sigma_{1}^{+} z^{n} \bar{z}^{\bar{n}}\left[\frac{\tau^{-1}}{1+\tau^{-2}}+\frac{i}{2} \ln \left(\frac{1+i \tau^{-1}}{1-i \tau^{-1}}\right)\right]+i \sigma_{2}^{+} z^{n} \bar{z}^{\bar{n}} \frac{\tau^{-2}}{1+\tau^{-2}}+ \\
& +\sigma_{1} z^{n} \bar{z}^{\bar{n}} \frac{i}{2} \ln \left(\frac{1+i \tau^{-1}}{1-i \tau^{-1}}\right) .
\end{aligned}
$$

Уравнение (Б.13) эквивалентно условиям на параметры $\sigma_{1}, \sigma_{1}^{+}$и $\sigma_{2}^{+}$

$$
\begin{gathered}
(2 n \bar{n}-n-\bar{n}) \sigma_{2}^{+}-(n-\bar{n}) \sigma_{1}=0, \\
2(n \bar{n}-n-\bar{n}+1) \sigma_{1}^{+}+i(n-\bar{n}) \sigma_{2}^{+}-i(n+\bar{n}-2) \sigma_{1}=0,
\end{gathered}
$$


если удовлетворены условия (5.2)-(5.4), обеспечивающие $\mathcal{D}$-замкнутость формы $\Phi_{2}^{n, \bar{n}}(y)$.

Используя (Б.1), (Б.10), (Б.14), (Б.16), (Б.18), (Б.20)-(Б.22), находим обшее решение системы (Б.4)-(Б.9) в виде (5.17)-(5.20), где $\sigma=i \sigma_{1}$.

Обратимся к случаю $\bar{n}=0, n<0$ (случай $n=0, \bar{n}<0$ может быть рассмотрен аналогично). Согласно таблице единственными параметрами, дающими ненулевой вклад в $\Phi_{1,2}^{n, \bar{n}}(y)$, являются $W_{1,2}, V_{1,2}$ и $Y_{1,2}$, а нетривиальными уравнениями в системе (Б.4)-(Б.9) являются (Б.5), (Б.7) и (Б.8). Аналогично случаю, рассмотренному вьше, фиксируем калибровку

$$
V_{1}=Y_{1}=0
$$

и получаем решение

$$
W_{1}(z, \bar{z}, \tau)=-\alpha_{Y}(n, 0) \frac{z^{n}}{\tau}+\sigma(n, 0) z^{n} \frac{\tau^{-1}}{1-i \tau^{-1}}
$$

Оставшийся случай $n, \bar{n}=0$ тривиален. Действительно, в этом случае единственные параметры, дающие вклад, - это $\alpha_{V}(0,0)$ и $V_{1}$. Параметр $\alpha_{V}(0,0)$ исчезает в силу уравнения (Б.7), а $V_{1}$ может быть откалиброван преобразованием (5.9).

\section{Список литературы}

[1] J. Maldacena. Adv. Theor. Math. Phys. 1998. V. 2. P. 231; hep-th/9711200.

[2] S. Ferrara, C. Fronsdal. Class. Q. Grav. 1998. V. 15. P. 2153; hep-th/9712239; M. Gunaydin, D. Minic. Nucl. Phys. B. 1998. V. 523. P. 145; hep-th/9802047; S. S. Gubser, I. R. Klebanov, A. M. Polyakov. Phys. Lett. B. 1998. V. 428. P. 105; hep-th/9802109.

[3] E. Witten. Adv. Theor. Math. Phys. 1998. V. 2. P. 253; hep-th/9802150.

[4] M. A. Vasiliev. Int. J. Mod. Phys. D. 1996. V. 5. P. 763.

[5] E. S. Fradkin, M. A. Vasiliev. Phys. Lett. B. 1987. V. 189. P. 89; Nucl. Phys. B. 1987. V. 291. P. 141.

[6] J. D. Brown, M. Henneaux. Commun. Math. Phys. 1986. V. 104. P. 207.

[7] M. A. Vasiliev. Fortschr. Phys. 1988. V. 36. P. 33.

[8] M. P. Blencowe. Class. Q. Grav. 1989. V. 6. P. 443.

[9] M. A. Vasiliev. Mod. Phys. Lett. A. 1992. V. 7. P. 3689.

[10] S. F. Prokushkin, M. A. Vasiliev. Nucl. Phys. B. 1999. V. 545. P. 385; hep-th/9806236.

[11] M. A. Vasiliev. Class. Q. Grav. 1994. V. 11. P. 649.

[12] M. A. Vasiliev. Fortschr. Phys. 1987. V. 35. Р. 741; М. А. Васильев. ЯФ. 1987. Т. 45. C. 1784 .

[13] F. A. Berends, G. J. H. Burgers, H. van Dam. Nucl. Phys. B. 1986. V. 271. P. 429.

[14] A. K. H. Bengtsson, I. Bengtsson. Phys. Lett. B. 1986. V. 174. P. 294.

[15] D. Anselmi. Nucl. Phys. B. 1999. V. 541. P. 323. 